\title{
Prediction, Tracking and Retrodiction for Path-Constrained Targets
}




\title{
PREDICTION, TRACKING AND RETRODICTION FOR PATH-CONSTRAINED TARGETS
}

BY

K. KRISHANTH, B.Sc.

\begin{abstract}
A THESIS
SUBMITTED TO THE DEPARTMENT OF ELECTRICAL \& COMPUTER ENGINEERING AND THE SCHOOL OF GRADUATE STUDIES

OF MCMASTER UNIVERSITY
\end{abstract}

IN PARTIAL FULFILMENT OF THE REQUIREMENTS

FOR THE DEGREE OF

Master of Applied Science

(c) Copyright by K. Krishanth, August 2012

All Rights Reserved 
Master of Applied Science (2012)

(Electrical \& Computer Engineering)
McMaster University

Hamilton, Ontario, Canada

TITLE: Prediction, Tracking and Retrodiction for PathConstrained Targets

AUTHOR:

K. Krishanth

B.Sc., (Electronic \& Telecommunication Engineering)

University of Moratuwa, Moratuwa, Sri Lanka

SUPERVISOR: $\quad$ Dr. T. Kirubarajan

NUMBER OF PAGES: xiii, 67 
To my Amma, Appa and Aunty 


\section{Abstract}

Prediction, tracking, and retrodiction for targets whose motion is constrained by external conditions (e.g., shipping lanes, roads) present many challenges to tracking systems. The targets are moving along a path, defined by way-points and segments. Measurements are obtained by sensors at low revisit rates (e.g., spaceborne). Existing tracking algorithms assume that the targets follow the same motion model between successive measurements, but in a low revisit rate scenario targets may change the motion model between successive measurements. A prediction algorithm is proposed here, which addresses this issue by considering possible motion model whenever targets move to a different segment. Further, when a target approaches a junction, it has the possibility to travel into one of the multiple segments connected to that junction. To predict the probable locations, multiple hypotheses for segments are introduced and a probability is calculated for each segment hypothesis. When measurements become available, segment hypothesis probability is updated based on a combined mode likelihood and a sequential probability ratio test is carried out to reject the hypotheses with low probability. Retrodiction for path constrained targets is also considered, because in some scenarios it is desirable to find out the target's exact location at some previous time (e.g., at the time of an oil leakage). A retrodiction

algorithm is developed for path constrained targets so as to facilitate motion forensic 
analysis. Simulation results are presented to validate the proposed algorithms.

KEYWORDS: prediction, target tracking, retrodiction, path-constrained targets, segment hypothesis 


\section{Acknowledgements}

The successful completion of my research presented in this thesis could not have been accomplished without the help and support of a number of people. I am glad to have the opportunity to acknowledge them here.

First and foremost, I would like to express my deepest gratitude to my academic supervisor, Dr. T. Kirubarajan whose expert advice and guidance, motivated me achieve excellence in my work. I would like to thank him for his valuable inputs throughout the course of my research.

I am most grateful to Dr. Tharmarasa, for his good advice and support. His thorough reviews and perceptive comments greatly improved the quality of my thesis work. I would also like to thank Dr. Alexandru Patriciu and Dr. Steve Hranilovic for being members of my thesis defense committee. I appreciate all the time the members of my committee took to read this thesis and for providing their input and thoughts on the subject.

I am thankful to the Department of Electrical \& Computer Engineering, for providing me an opportunity to pursue my research at McMaster University. I would also like to thank Cheryl Gies of the Department of Electrical \& Computer Engineering for providing me with prompt administrative support.

Last, but by no means least, I would like to thank my parents, aunty, sisters and 
their families for their their unequivocal support throughout, as always, for which my mere expression of thanks likewise does not suffice. 


\section{Notation and abbreviations}

\section{Abbreviations}

AIS Automatic Identification System

FIM Fisher Information Matrix

GMTI Ground Moving Target Indicator

IMM Interacting Multiple Model

PCRLB Posterior Cramer Rao lower bound

RMSE Root Mean Square Error

VS-MM Variable Structure Multiple Model

VS-IMM Variable Structure Interacting Multiple Model 


\section{Notations}

E Expectation

$W($.$) \quad Filter gain$

$J \quad$ Fisher information matrix

h(.) Hypothesis

$\Psi($.$) \quad Hypotheses set$

$A($.$) \quad IMM mode set$

$v($.$) \quad Innovation$

$S($.$) \quad Innovation covariance$

$M V \quad$ Maximum velocity

$\Sigma($.$) \quad Measurement noise covariance$

$H($.$) Observation matrix$

$k \quad$ Present time step

$\sigma_{a} \quad$ Process noise along the path

$\Sigma($.$) \quad Process noise covariance$

$\sigma_{o} \quad$ Process noise orthogonal to the path

$R($.$) \quad Rotation matrix$

A Retrodiction gain

$S_{k} \quad$ Segment in present time

$F($.$) \quad State transition matrix$

M. Target motion mode

$[.]^{T} \quad$ Transpose of a matrix

$r \quad$ Velocity ratio

$\Gamma($.$) \quad Vector gain$

$w(k) \quad$ White Gaussian measurement noise

$v($.$) \quad White Gaussian process noise ix$ 


\section{Contents}

Abstract $\quad$ iv

Acknowledgements $\quad$ vi

Notation and abbreviations viii

1 Introduction and Problem Statement 1

1.0.1 Prediction and Tracking ............... 1

1.0.2 Retrodiction . . . . . . . . . . . . . . 3

1.1 Estimator for Target Tracking . . . . . . . . . . . . . . . . 4

1.1.1 Kalman Filter . . . . . . . . . . . . . . . . . . 5

1.1.2 Interacting Multiple Model Estimator . . . . . . . . . . . . 7

1.2 Motivation and Contribution of the Thesis . . . . . . . . . . . . 10

1.3 Organization of the Thesis . . . . . . . . . . . . 11

1.4 Related Publications . . . . . . . . . . . . . . . . . . 12

2 Path Constrained Estimator 13

2.1 Path Constrained Estimator Algorithm . . . . . . . . . . . . 13

2.1.1 Validation Test for Mode Set Adaptation . . . . . . . . . . . . 20 
2.1.2 Directional Process Noise . . . . . . . . . . . . . . . . . . 21

2.1.3 Velocity Prediction During Segment Changes . . . . . . . . . 22

2.1.4 Hypothesis Generation Based on Segments . . . . . . . . . 23

3 Retrodiction for Path Constrained Targets 25

3.1 General Retrodiction Algorithm . . . . . . . . . . . . . 26

3.2 Path Constrained Retrodiction Algorithm _. . . . . . . . 28

3.3 Posterior Cramer Rao Lower Bound for Path Constrained Targets . . 31

4 Simulation Studies and Results $\quad 34$

4.1 Simulation Test . . . . . . . . . . . . . . . . . . . . 34

4.2 Real Data Test . . . . . . . . . . . . . . . . 46

5 Conclusions and Future Work $\quad 54$

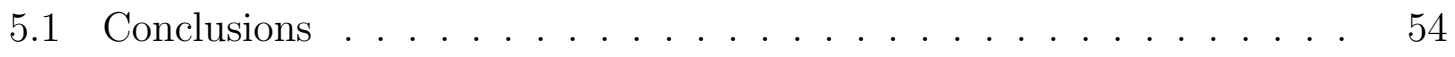

5.2 Future Work . . . . . . . . . . . . . . . . . . . 55

$\begin{array}{ll}\text { A Algorithm Derivations } & 56\end{array}$

A.0.1 Filtering Algorithm Derivation . . . . . . . . . . 56

A.0.2 Retrodiction Algorithm Derivation _. . . . . . . 58

A.0.3 Retrodiction Algorithm Derivation for Path Constrained Targets 62 


\section{List of Figures}

1.1 IMM estimator (one cycle). . . . . . . . . . . . . . . . . . 10

2.1 Mode changes at time $k-\tau$ due to segment change . . . . . . . . . 14

2.2 Path constrained filtering (one cycle) . . . . . . . . . . . 19

2.3 Directional process noise for path constrained targets . . . . . . . . . 21

2.4 Segment hypothesis tree . . . . . . . . . . . . . . . 24

3.1 Mode changes at time $t+\delta$ due to segment change . . . . . . . . 29

4.1 Path map for simulation . . . . . . . . . . . . . . 36

4.2 Target dynamics variation . . . . . . . . . . . . . . 37

4.3 RMS position errors for estimators . . . . . . . . . . . . . . . 39

4.4 RMS velocity errors for estimators . . . . . . . . . . . . . . . . . 40

4.5 RMS position errors for retrodiction . . . . . . . . . . . . . 41

4.6 RMS velocity errors for retrodiction . . . . . . . . . . . . . . . 42

4.7 RMS position errors comparison for proposed algorithms . . . . . . 43

4.8 RMS velocity errors comparison for proposed algorithms . . . . . . . 44

4.9 PCRLB position comparison . . . . . . . . . . . . 45

4.10 Location (Google maps) . . . . . . . . . . . . . . . . 47

4.11 Whole measurements plot . . . . . . . . . . . . . . 48

4.12 Path map with segments and way points . . . . . . . . . . . 49 
4.13 Tracks from 25 targets . . . . . . . . . . . . . . 50

4.14 Tracks from prediction . . . . . . . . . . . . . . 51

4.15 Tracks from retrodiction . . . . . . . . . . . . . . 52

4.16 RMS position errors comparison . . . . . . . . . . . . . 53 


\section{Chapter 1}

\section{Introduction and Problem}

\section{Statement}

The targets are moving along a constrained path with varying terrain conditions. The terrain conditions can affect the movement of targets in direction, velocity and maneuvering. The path is defined by way-points and segments (Kirubarajan et al., 2000). Further, a path can branch, merge or cross. Because of the varying terrain and path constraints, target trajectory can evolve according to one of the multiple motion modes available. It is evident that a multiple model approach is required to handle all possible motion modes and path constraints.

\subsubsection{Prediction and Tracking}

Fixed structure Interacting Multiple Model (IMM) estimator (Blom and Bar-Shalom, 1988) consists of models to handle all possible motion modes and path constraints can be a solution to handle all possible motion modes. But due to the "competition" 
among the models (Li and Bar-Shalom, 1996), fixed structure IMM results in a degraded estimates. This issue is overcome by Variable Structure Interacting Multiple Model (VS-IMM) filter, which adaptively modifies the filter modules based on terrain topography. It is a modified version of IMM where models in a mode set can vary based on constraints. Since the mode set is adjusted based on the path map information available, VS-IMM estimator outperforms typical IMM estimator (Kirubarajan et al., 2000) and the effectiveness of VS-IMM for path constrained targets has been shown in (Kirubarajan et al., 2000), (Pannetier et al., 2005) and (Shea et al., 2000). In (Streller, 2008), Variable Structure Multiple Model (VS-MM) estimator, another version of multiple model approach, which does not use interaction like VS-IMM, is used to track ground targets effectively. By avoiding interaction between the multiple models, VS-MM avoids the bad estimations given by VS-IMM at junctions (Streller, 2008). Particle filter approach is also widely used for ground targets with path maps and has shown better performances in (Arulampalam et al., 2002), (Kyriakides et al., 2008) and (Ulmke and Koch, 2006). But due to the large computation time associated with the particle filter, Kalman filter approaches (IMM, VS-MM, VS-IMM) are widely used for the path constrained targets (Streller, 2008).

Since the targets are constrained to path, prediction should be along the path with the available prior information (e.g., path map, target class, destination). Existing algorithms, except (Pannetier et al., 2005), do not consider path map information in prediction stage, which results in predicted target state in an off-path position. In (Pannetier et al., 2005), a projection mechanism is used to constrain the target state to be on path. In this thesis, a different approach is used to keep the predicted state along the path rather than the estimate. Further when a target moves towards a 
junction, the path on which the target may travel is unknown. In (Kirubarajan et al., 2000) and (Shea et al., 2000), uncertainty at junctions is handled by temporarily augmenting the mode set to accommodate all possible modes, which represent the target motion along the possible segments. In this approach, the segment followed by the target is not considered. In this thesis junctions are handled by multiple hypotheses for segments (Pannetier et al., 2005). Segment hypotheses keep all the possible path sequences a target can travel and when the measurements are received, a sequential probability ratio test is carried out to select the best hypothesis and reject all other hypotheses. In addition, magnitude of target velocity may vary based on the path segment. Existing algorithms do not have the mechanism to incorporate this prior information. A prediction algorithm is proposed in this thesis which follows a multi-step prediction technique and incorporates the magnitude and direction of target velocity constrained to path segment.

\subsubsection{Retrodiction}

Smoothing or retrodiction is the estimation of the state at a time within the data time interval (Bar-Shalom et al., 2001). With a certain time delay and some additional computational load, the estimate at a given time can be improved significantly. Retrodiction can be used for performance evaluation for real time filters and motion forensic analysis where small time delay is permitted. Various methods have been proposed for retrodiction in literature. Fixed interval smoothing, delaying certain amount of time to get a better estimate, for Markovian switching systems is developed in (Helmick et al., 1995). Two multiple model filters, where one of the filters propagates in the forward-time direction and the other one propagates in the 
backward-time direction is used in the above smoothing algorithm. This is similar to the Fraser-Potter (Fraser and Potter, 1969) adopted for single model smoothing algorithm. In (Koch, 2000), a fixed interval smoothing to IMM-MHT applications is given based on standard Rauch-Tung-Striebel (RTS) smoothing (Rauch et al., 1965). Fixed lag smoothing uses the past and present data to compute the state estimate certain time step behind the present time. In (Helmick et al., 1994), two different approaches for one step fixed lag smoothing algorithms are given where the methods differ by the sampling period upon which the state of the system is conditioned. An augmented approach, applying the basic IMM approach to a state augmented system, for fixed lag IMM smoothing is given in (Tugnait, 2000). But no algorithms have been specifically developed for path constrained problems in literature. In this thesis a retrodiction algorithm is presented for variable structure IMM based on (Koch, 2000) and then it is extended to path constrained targets.

\subsection{Estimator for Target Tracking}

Due to the large computation time associated with the particle filter method, Kalman filter approaches are widely used for path constrained target tracking applications (Streller, 2008). Subsection 1.1.1 contains the equations for a Kalman filter and subsection 1.1.2 presents the equations for IMM filter, which are important for a better understanding of this thesis. 


\subsubsection{Kalman Filter}

The target evolution state $\mathbf{x}(k)$, which was defined in (Bar-Shalom et al., 2001), can be written as

$$
\mathbf{x}(k)=F(k, k-1) \mathbf{x}(k-1)+\Gamma(k, k-1) v(k-1)
$$

where $F(k, k-1)$ is the state transition matrix and $\Gamma(k, k-1)$ is the vector gain. $v(k-$ $1)$ is the white Gaussian process noise sequence with covariance given by $Q(k, k-1)$. Target originated measurement can be written as

$$
\mathbf{z}(k)=H(k) \mathbf{x}(k)+w(k)
$$

where $H(k)$ is the observation matrix. The white Gaussian measurement noise sequence $w(k)$ is independent of $v(k)$ and its covariance is $\Sigma(k)$.

The predicted state $\hat{x}(k \mid k-1)$ at time $k$ is

$$
\hat{x}(k \mid k-1)=F(k, k-1) \hat{x}(k-1 \mid k-1)
$$

and the associated predicted state covariance $P(k \mid k-1)$ is

$P(k \mid k-1)=F(k, k-1) P(k-1 \mid k-1) F(k, k-1)^{\prime}+\Gamma(k, k-1) Q(k, k-1) \Gamma(k, k-1)^{T}$

where $\hat{x}(k-1 \mid k-1)$ is the state estimate and $P(k-1 \mid k-1)$ is the associated covariance 
at time $(k-1)$. The predicted measurement $\hat{z}(k \mid k-1)$ at time $k$ is

$$
\hat{z}(k \mid k-1)=H(k) \hat{x}(k \mid k-1)
$$

and the associated innovation covariance is

$$
S(k)=H(k) P(k \mid k-1) H(k)^{\prime}+R(k)
$$

The state estimate update at time $k$ is

$$
\hat{x}(k-1 \mid k-1)=\hat{x}(k \mid k-1)+W(k) v(k)
$$

where $W(k)$ is the filter gain given as

$$
W(k)=P(k \mid k-1) H(k)^{\prime} S(k)^{-1}
$$

and innovation is given by

$$
v(k)=z(k)-\hat{z}(k \mid k-1)
$$

The covariance matrix associated with $\hat{x}(k-1 \mid k-1)$ is given by

$$
P(k)=P(k \mid k-1)-W(k) S(k) W(k)^{\prime}
$$

The above equations are provided here for completeness and to introduce the notions for later use. 


\subsubsection{Interacting Multiple Model Estimator}

In IMM estimator, state estimate is computed under each possible current model using available fixed filters with each filter using a different 'mixed initial condition'. For each filter, 'mixed initial condition' is calculated using the previous mode conditioned estimates. The detailed derivation for IMM estimator is given in (Blom and Bar-Shalom, 1988) and steps required to calculate the state estimate is given here.

Let $A(k-1)=\left(M_{1}(k-1), \ldots, M_{i}(k-1), \ldots\right)$ be the mode set of the IMM estimator in the time interval $(k-2, k-1]$ and $A(k)=\left(M_{1}(k), \ldots, M_{j}(k), \ldots\right)$ be the mode set in the time interval $(k-1, k]$.

\section{Step 1 - Calculation of the mixing probabilities}

The probability that mode $M_{i}$ was in effect at $(k-1)$ given that mode $M_{j}$ is in effect at $k$ conditioned on $Z_{1}^{k-1}$ is

$$
\begin{aligned}
\mu_{i \mid j}(k-1 \mid k-1) & =P\left\{M_{i} \mid M_{j}, Z_{1}^{k-1}\right\} \\
& =\frac{1}{C_{j}} P\left\{M_{j} \mid M_{i}, Z_{1}^{k-1}\right\} P\left\{M_{i} \mid Z_{1}^{k-1}\right\}
\end{aligned}
$$

The above mixing probabilities can be written as

$$
\mu_{i \mid j}(k-1 \mid k-1)=\frac{1}{C_{j}}\left[p_{i j}\left(S_{k-1}\right)\right] \mu_{i}(k-1) \quad \forall i, \forall j
$$


where the normalizing constants are given by

$$
C_{j}=\sum_{M_{i} \in A(k-1)}\left[p_{i j}\left(S_{k-1}\right)\right] \mu_{i}(k-1) \quad \forall j
$$

\section{Step 2 - IMM Mixing}

Mixed initial condition for the filter matched to $M_{j}(k-\tau)$ is computed as

$$
\hat{x}^{0 j}(k-1 \mid k-1)=\sum_{M_{i} \in A(k-1)} \hat{x}^{i}(k-1 \mid k-1) \mu_{i \mid j}(k-1 \mid k-1) \quad \forall j
$$

The corresponding covariance is given by

$$
\begin{aligned}
P^{0 j}(k-1 \mid k-1) & =\sum_{M_{i} \in A(k-1)} \mu_{i \mid j}(k-1 \mid k-1)\left\{\hat{x}^{i}(k-1 \mid k-1)\right. \\
& +\left[\hat{x}^{i}(k-1 \mid k-1)-\hat{x}^{0 j}(k-1 \mid k-1)\right] \\
& {\left.\left[\hat{x}^{i}(k-1 \mid k-1)-\hat{x}^{0 j}(k-1 \mid k-1)\right]^{T}\right\} \quad \forall j }
\end{aligned}
$$

\section{Step 3 - Mode Matched Filtering}

The estimate $\hat{x}^{0 j}(k-1 \mid k-1)$ and covariance $P^{j}(k-1 \mid k-1)$ are used as input to the

filter matched to $M_{j}$, which uses $z(k)$ to yield $\hat{x}^{j}(k \mid k)$ and $\hat{P}^{j}(k \mid k)$. The likelihood function corresponding to the above filtering processes are calculated by

$$
\Lambda_{j}(k)=P\left[z(k) \mid M_{j}, Z_{1}^{k-1}\right] \quad \forall j
$$

\section{Step 4 - Mode probability updating}

The probability of model $\left(M_{j}\right)$ being in effect during the time interval $(k, k-1]$ given 
measurement data up to $k$ can be calculated as

$$
\begin{aligned}
\mu_{j}(k \mid k) & =P\left\{M_{j} \mid Z_{1}^{k}\right\} \\
& =\frac{1}{C} P\left[z(k) \mid M_{j}, Z_{1}^{k-1}\right] P\left[M_{j} \mid Z_{1}^{k-1}\right] \\
& =\frac{1}{C} \Lambda_{j}(k) \mu_{j}(k \mid k-1)
\end{aligned}
$$

where $\mu_{j}(k \mid k-1)$ can be written as

$$
\mu_{j}(k \mid k-1)=\sum_{M_{i} \in A(k-1)}\left[p_{i j}\left(S_{k-1}\right)\right] \mu_{i}(k-1)
$$

\section{Step 5 - State estimate and covariance combination}

The final state estimate and covariance are

$$
\begin{aligned}
\hat{x}(k \mid k) & =\sum_{M_{j} \in A(k)} \hat{x}^{j}(k \mid k) \mu_{j}(k \mid k) \\
P(k \mid k) & =\sum_{M_{j} \in A(k)} \mu_{j}(k \mid k)\left\{P^{j}(k \mid k)+\left[\hat{x}^{j}(k \mid k)-\hat{x}(k \mid k)\right]\left[\hat{x}^{j}(k \mid k)-\hat{x}(k \mid k)\right]^{T}\right\}
\end{aligned}
$$

Figure 1.1 describes the IMM algorithm that consists of two interacting filters. 

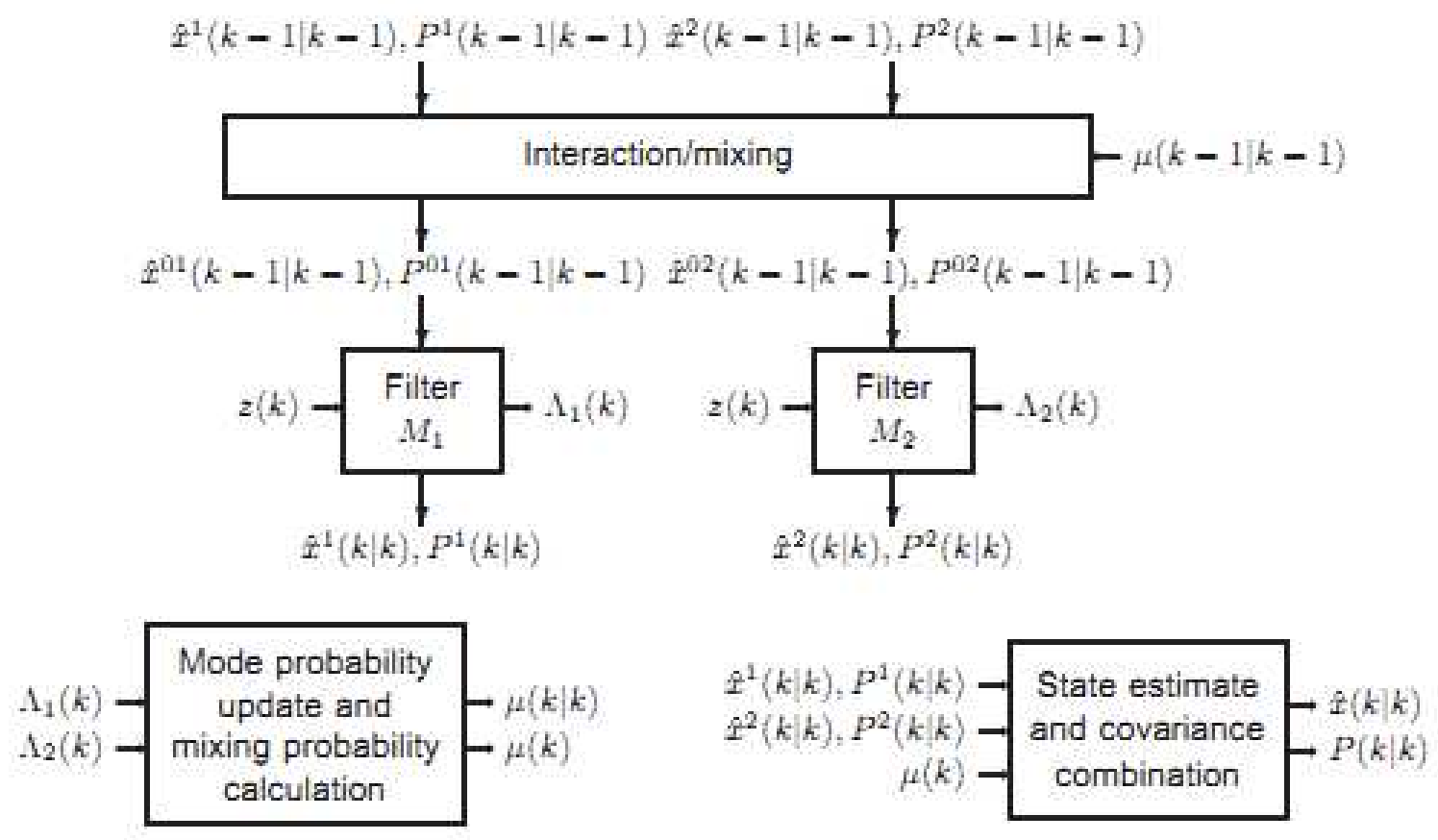

Figure 1.1: IMM estimator (one cycle).

\subsection{Motivation and Contribution of the Thesis}

This thesis addresses the problem of prediction, tracking and retrodiction of path constrained targets where measurements are obtained by sensors with low revisit rates. For example, a spaceborne sensor, with varying measurement intervals may take up to 12 hours to update a measurement related to a target. The high measurement interval time between successive measurements will cause potentially degraded performance during prediction in already existing algorithms (Kirubarajan et al., 2000), (Pannetier et al., 2005). Updating the mode set upon a receival of a measurement is the process carried out in the above algorithms. But in a scenario where measurements are obtained by sensors at low revisit rates, mode set may need to be updated 
during the measurement interval due to a change in the segment in which the target is moving. The proposed algorithm addresses this issue in prediction by considering the time at which the target moves to a different segment instead of waiting for the measurement to be received. In addition, segment hypotheses are used to handle the motion uncertainty of the path constrained targets in the intersections. Retrodiction for path constrained targets is not available in the literature and a novel retrodiction algorithm is presented in this thesis. The retrodiction algorithm considers path map information and retrodiction gain is derived considering the path constraints imposed. To evaluate the performance of the proposed algorithms, Posterior Cramer Rao lower bound (PCRLB) for path constrained targets is given.

\subsection{Organization of the Thesis}

Subsequent chapters will explain the algorithms derived for prediction, tracking and retrodiction in detail. In Chapter 2 derived estimator for path constrained targets is given. It also consists of the techniques used to improve the prediction and tracking algorithm. Chapter 3 poses the retrodiction algorithm derived for path constrained targets where initially a retrodiction algorithm is derived and then later it is extended to path constrained targets. PCRLB for path constrained targets is given in 3.3. Simulation tests and results are given in Chapter 4 where one test is carried out in a simulated scenario and another test is carried out on real data gathered from exactEarth Inc. The author's conclusions are presented in Chapter 5 along with a discussion on possible future research directions. 


\subsection{Related Publications}

- K. Krishanth, R. Tharmarasa, T. Kirubarajan, P. Valin and E. Meger, "Prediction, tracking, and retrodiction for path-constrained targets, " Proc. of the Signal and Data Processing of Small Targets, Baltimore, MD, May 2011.

- K. Krishanth, R. Tharmarasa, T. Kirubarajan, P. Valin and E. Meger, "Prediction, tracking, and retrodiction for path-constrained targets, " submitted to IEEE Transactions on Aerospace and Electronic Systems Journal. 


\section{Chapter 2}

\section{Path Constrained Estimator}

Variable Structure Interacting Multiple Model (VS-IMM) estimator is developed based on IMM algorithm. But models in the mode set are fixed in IMM but it can vary based on constraints in VS-IMM. The proposed path constrained estimator developed in this research is extended on VS-IMM algorithm with path map information.

\subsection{Path Constrained Estimator Algorithm}

Let $S_{k-1}$ be the segment in effect, $A(k-1)=\left(M_{1}(k-1), M_{2}(k-1), \ldots, M_{i}(k-1), \ldots\right)$ be the mode set of the IMM estimator in the time interval $(k-2, k-1]$. Further $S_{k}$ is the segment in effect during the time interval $(k-1, k]$ and the mode set of the IMM estimator is $A(k)$, which may or may not include the models from $A(k-$ 1). Measurements are obtained by sensors with low revisit rates. In this scenario, the target may change from one to another segment within successively received measurements. Figure 2.1 shows that during the time interval $(k-1, k]$, target is 
in two different segments. Proposed algorithm considers this issue and predicts the target state more precisely. Let $(k-\tau)$ be the time at which the target changes the segment where $0<\tau<1$.

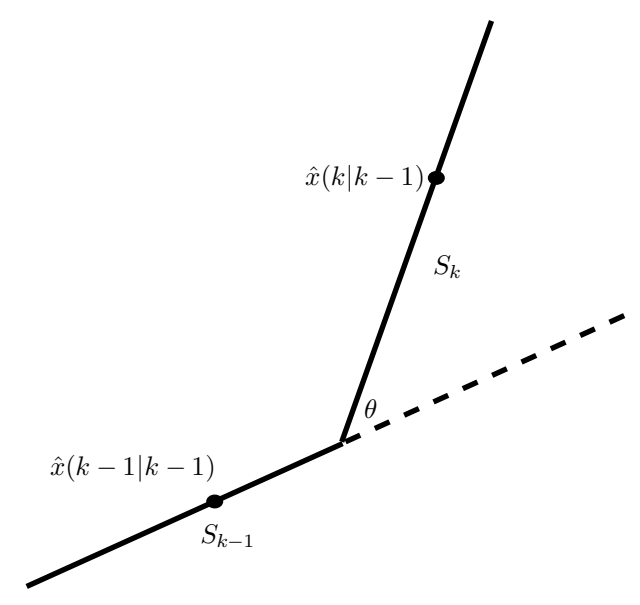

Figure 2.1: Mode changes at time $k-\tau$ due to segment change

The steps of the tracking algorithm are:

\section{Step 1 - Calculation of the mixing probabilities}

The probability that mode $M_{i}$ was in effect at $(k-1)$ given that mode $M_{i^{\prime}}$ is in effect at $(k-\tau)$ conditioned on $Z_{1}^{k-1}$ is

$$
\begin{aligned}
\mu_{i \mid i^{\prime}}(k-1 \mid k-1) & =P\left\{M_{i} \mid M_{i^{\prime}}, Z_{1}^{k-1}\right\} \\
& =\frac{1}{C_{i^{\prime}}} P\left\{M_{i^{\prime}} \mid M_{i}, Z_{1}^{k-1}\right\} P\left\{M_{i} \mid Z_{1}^{k-1}\right\}
\end{aligned}
$$


The above mixing probabilities can be written as

$$
\mu_{i \mid i^{\prime}}(k-1 \mid k-1)=\frac{1}{C_{i^{\prime}}}\left[p_{i i^{\prime}}\left(S_{k-1}\right)\right] \mu_{i}(k-1) \quad \forall i, \forall i^{\prime}
$$

where the normalizing constants are given by

$$
C_{i^{\prime}}=\sum_{M_{i} \in A(k-1)}\left[p_{i i^{\prime}}\left(S_{k-1}\right)\right] \mu_{i}(k-1) \quad \forall i^{\prime}
$$

\section{Step 2 - IMM Mixing}

Mixed initial condition for the filter matched to $M_{i^{\prime}}(k-\tau)$ is computed as

$$
\hat{x}^{0 i^{\prime}}(k-1 \mid k-1)=\sum_{M_{i} \in A(k-1)} \hat{x}^{i}(k-1 \mid k-1) \mu_{i \mid i^{\prime}}(k-1 \mid k-1) \quad \forall i^{\prime}
$$

The corresponding covariance is given by

$$
\begin{aligned}
P^{0 i^{\prime}}(k-1 \mid k-1) & =\sum_{M_{i} \in A(k-1)} \mu_{i \mid i^{\prime}}(k-1 \mid k-1)\left\{\hat{x}^{i}(k-1 \mid k-1)\right. \\
& +\left[\hat{x}^{i}(k-1 \mid k-1)-\hat{x}^{0 i^{\prime}}(k-1 \mid k-1)\right] \\
& {\left.\left[\hat{x}^{i}(k-1 \mid k-1)-\hat{x}^{0 i^{\prime}}(k-1 \mid k-1)\right]^{T}\right\} \quad \forall i^{\prime} }
\end{aligned}
$$

\section{Step 3 - Mode Matched Filtering}

The estimate $\hat{x}^{0 i^{\prime}}(k-1 \mid k-1)$ and covariance $P^{0 i^{\prime}}(k-1 \mid k-1)$ are used as input to the filter matched to $M_{i^{\prime}}$ to derive predicted state $\hat{x}^{i^{\prime}}(k-\tau \mid k-1)$ and covariance 
$P^{i^{\prime}}(k-\tau \mid k-1)$.

$$
p\left\{x(k-\tau) \mid M_{i^{\prime}}, Z_{1}^{k-1}\right\}=\mathcal{N}\left[x(k-\tau) ; E\left[x(k-\tau) \mid M_{i^{\prime}}, \hat{x}^{0 i^{\prime}}(k-1 \mid k-1)\right], \operatorname{cov}[.]\right]
$$

This gives

$$
\begin{aligned}
\hat{x}^{i^{\prime}}(k-\tau \mid k-1) & =F^{i^{\prime}}(k-\tau, k-1) \hat{x}^{0 i^{\prime}}(k-1 \mid k-1) \\
\hat{P}^{i^{\prime}}(k-\tau \mid k-1) & =F^{i^{\prime}}(k-\tau, k-1) \hat{P}^{0 i^{\prime}}(k-1 \mid k-1) F^{i^{\prime}}(k-\tau, k-1)^{T} \\
& +Q^{i^{\prime}}(k-\tau, k-1)
\end{aligned}
$$

The predicted state $\hat{x}^{i^{\prime}}(k-\tau \mid k-1)$ is fed to the filter matched to $M_{j^{\prime}}$ to compute the predicted state $\hat{x}^{i^{\prime} j^{\prime}}(k \mid k-1)$ and covariance $P^{i^{\prime} j^{\prime}}(k \mid k-1)$.

$$
\begin{aligned}
p\left[x(k) \mid M_{j^{\prime}}, M_{i^{\prime}}, Z_{1}^{k-1}\right] & =P\left[x(k) \mid x(k-\tau), M_{j^{\prime}}, M_{i^{\prime}}, Z_{1}^{k-1}\right] P\left[x(k-\tau) \mid M_{j^{\prime}}, M_{i^{\prime}}, Z_{1}^{k-1}\right] \\
& =P\left[x(k) \mid x(k-\tau), M_{j^{\prime}}, Z_{1}^{k-1}\right] P\left[x(k-\tau) \mid M_{i^{\prime}}, Z_{1}^{k-1}\right]
\end{aligned}
$$

Since there is a change in the direction of velocity at time $(k-\tau)$ due to segment change, it has to be considered during the above derivation as well. A rotation matrix is used for this purpose. For e.g., in a two dimensional tracking scenario with target state $\mathbf{x}(k)$ defined by the 4-dimensional vector $\mathbf{x}(k)=\left[\begin{array}{llll}x(k) & \dot{x}(k) & y(k) & \dot{y}(k)\end{array}\right]^{\prime}$ 
rotation matrix is given by

$$
R=\left[\begin{array}{cccc}
1 & 0 & 0 & 0 \\
0 & \cos \theta & 0 & -\sin \theta \\
0 & 0 & 1 & 0 \\
0 & \sin \theta & 0 & \cos \theta
\end{array}\right]
$$

where $\theta$ is the angle between the two segments in counter clockwise direction as shown in Figure 2.1.

Applying Gaussian distribution computation rules to (2.9) and incorporating the rotation matrix $(R)$ gives the following equations for $\hat{x}^{i^{\prime} j^{\prime}}(k \mid k-1)$ and $\hat{P}^{i^{\prime} j^{\prime}}(k \mid k-1)$

$$
\begin{aligned}
\hat{x}^{i^{\prime} j^{\prime}}(k \mid k-1) & =F^{j^{\prime}}(k, k-\tau) R F^{i^{\prime}}(k-\tau, k-1) \hat{x}^{0 i^{\prime}}(k-1 \mid k-1) \\
\hat{P}^{i^{\prime} j^{\prime}}(k \mid k-1) & =F^{j^{\prime}}(k, k-\tau) R\left\{F^{i^{\prime}}(k-\tau, k-1) \hat{P}^{0 i^{\prime}}(k-1 \mid k-1) F^{i^{\prime}}(k-\tau, k-1)^{T}\right. \\
& \left.+Q^{i^{\prime}}(k-\tau, k-1)\right\} R^{T} F^{j^{\prime}}(k, k-\tau)^{T}+Q^{j^{\prime}}(k, k-\tau)
\end{aligned}
$$

When measurements become available, updated state $\hat{x}^{i^{\prime} j^{\prime}}(k \mid k)$ and covariance $P^{i^{\prime} j^{\prime}}(k \mid k)$ are computed in a standard IMM method and the likelihood function corresponding to the above filtering processes are calculated by

$$
\Lambda_{i^{\prime} j^{\prime}}(k)=P\left[z(k) \mid M_{j^{\prime}}, M_{i^{\prime}}, Z_{1}^{k-1}\right] \quad \forall i^{\prime}, \forall j^{\prime}
$$

\section{Step 4 - Mode probability updating}

The probability of model $\left(M_{i^{\prime}}, M_{j^{\prime}}\right)$ being in effect during the time interval $(k, k-1]$ 
given measurement data up to $k$ can be calculated as

$$
\begin{aligned}
\mu_{i^{\prime} j^{\prime}}(k \mid k) & =P\left\{M_{j^{\prime}}, M_{i^{\prime}} \mid Z_{1}^{k}\right\} \\
& =P\left\{M_{j^{\prime}}, M_{i^{\prime}} \mid z(k), Z_{1}^{k-1}\right\} \\
& =\frac{1}{C_{i^{\prime} j^{\prime}}} P\left[z(k) \mid M_{j^{\prime}}, M_{i^{\prime}}, Z_{1}^{k-1}\right] P\left[M_{j^{\prime}} \mid M_{i}^{\prime}, Z_{1}^{k-1}\right] P\left[M_{i}^{\prime} \mid Z_{1}^{k-1}\right] \\
& =\frac{1}{C_{i^{\prime} j^{\prime}}} \Lambda_{i^{\prime} j^{\prime}}(k)\left[p_{i^{\prime} j^{\prime}}\left(S_{k-1}, S_{k}\right)\right] \mu_{i^{\prime}}(k-\tau)
\end{aligned}
$$

where $\mu_{i^{\prime}}(k-\tau)$ are

$$
\begin{aligned}
\mu_{i^{\prime}}(k-\tau) & =P\left\{M_{i^{\prime}} \mid Z_{1}^{k-1}\right\} \\
& =\sum_{M_{i} \in A(k-1)} P\left\{M_{i^{\prime}} \mid M_{i}(k-1), Z_{1}^{k-1}\right\} P\left\{M_{i} \mid Z_{1}^{k-1}\right\} \\
& =\sum_{M_{i} \in A(k-1)}\left[p_{i i^{\prime}}\left(S_{k-1}\right)\right] \mu_{i}(k-1)
\end{aligned}
$$

\section{Step 5 - State estimate and covariance combination}

The final state estimate and covariance are

$$
\begin{aligned}
\hat{x}(k \mid k) & =\sum_{M_{j^{\prime}}, M_{i^{\prime}} \in A(k)} \hat{x}^{i^{\prime} j^{\prime}}(k \mid k) \mu_{i^{\prime} j^{\prime}}(k \mid k) \\
P(k \mid k) & =\sum_{M_{j^{\prime}}, M_{i^{\prime}} \in A(k)} \mu_{i^{\prime} j^{\prime}}(k \mid k)\left\{P^{i^{\prime} j^{\prime}}(k \mid k)+\left[\hat{x}^{i^{\prime} j^{\prime}}(k \mid k)-\hat{x}(k \mid k)\right]\left[\hat{x}^{\prime} j^{\prime}(k \mid k)-\hat{x}(k \mid k)\right]^{T}\right\}
\end{aligned}
$$



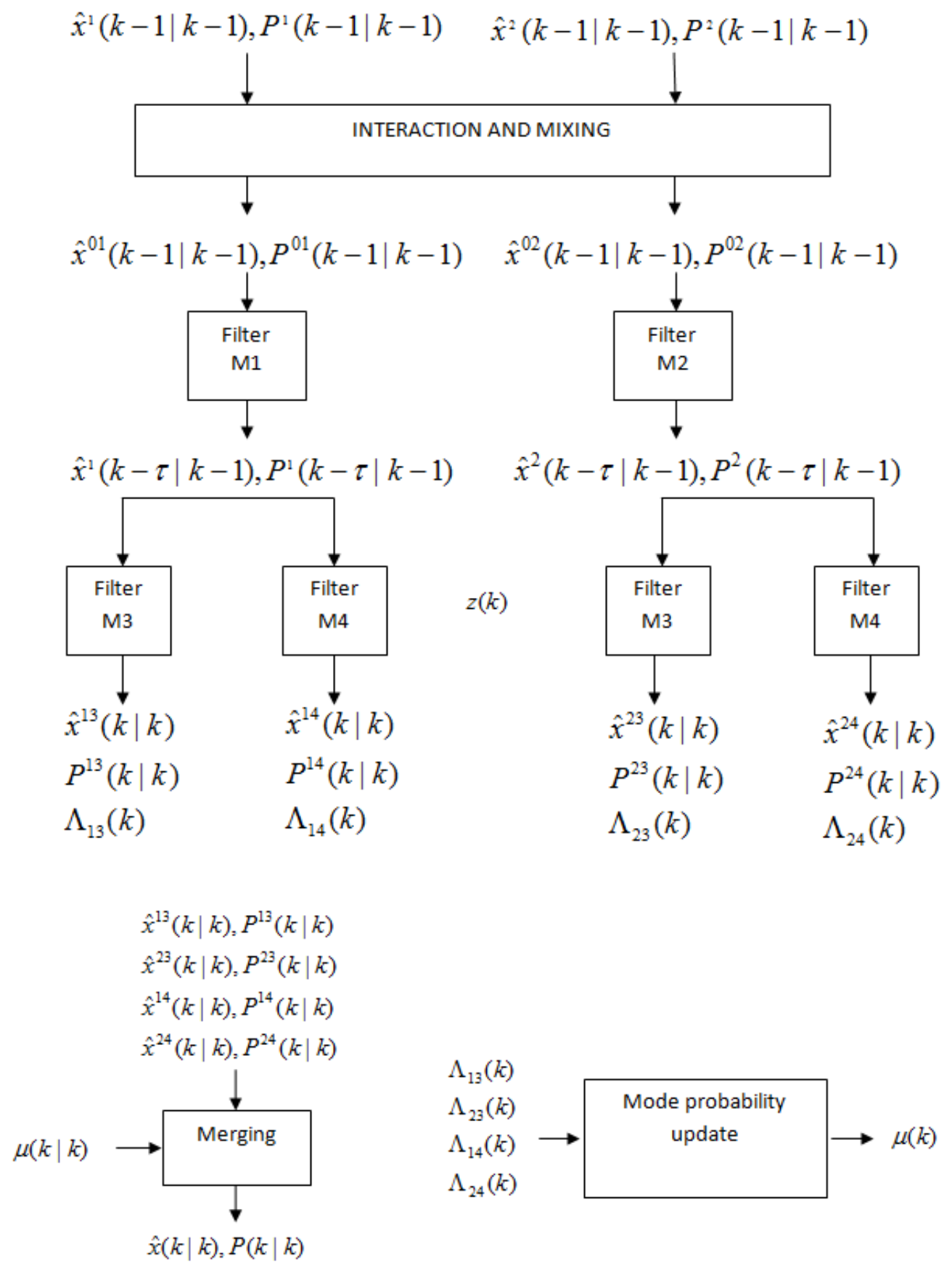

Figure 2.2: Path constrained filtering (one cycle) 


\subsubsection{Validation Test for Mode Set Adaptation}

At each scan $k$, it is required to find out the possible segments and corresponding models to be added to the mode set for a target. A validation region test (Kirubarajan et al., 2000) has to be carried out for this purpose. Since there are more than one mode in the mode set $A(k-1)$, there are more than one predicted state estimate and covariance, i.e., no common predicted state estimate or covariance. To solve this, predicted state estimate and predicted covariance correspond to the largest determinant among the modules in $A(k-1)$ is selected (Bar-Shalom and Li, 1995).

While carrying out the above test, another test is carried out for junctions. When a junction falls within the validation region, segment hypotheses (Section 2.1.4) are generated for each segment that falls within the validation region. A mode set is kept for each segment hypothesis and models corresponding to the segment is added to the respective mode set. But the problem with the above validation region is that there are instances where none of the junction falls within the validation region although the target is predicted to pass a junction. To overcome this problem, when none of the junction falls within the validation region, a test is carried out to check whether the predicted state falls outside the present segment or not. If it is still in the present segment, mode set will be kept as it is. But if it is not in the present segment, a new segment hypothesis is created for each segment connected with the present segment and updated with corresponding models. 


\subsubsection{Directional Process Noise}

Since the targets are constrained to move along the path, uncertainty along the path is higher compared to the uncertainty orthogonal to the path. The concept of "directional process noise" (Bar-Shalom and Li, 1995) is used to handle the above mentioned issue. Figure 2.3 shows the directional process noise concept corresponding to the path constrained targets. The estimation is carried out in X-Y coordinate system, so the directional process noise along and orthogonal to the path has to be converted into a covariance matrix $Q$ in $\mathrm{X}-\mathrm{Y}$ frame. Let standard deviation of process noise along the path be $\sigma_{a}$ and standard deviation in the orthogonal direction be $\sigma_{o}$. The conversion is done via (Yeddanapudi et al., 1997)

$$
Q=\left[\begin{array}{cc}
-\cos \psi & \sin \psi \\
\sin \psi & \cos \psi
\end{array}\right]\left[\begin{array}{cc}
\sigma_{o}^{2} & 0 \\
0 & \sigma_{a}^{2}
\end{array}\right]\left[\begin{array}{cc}
-\cos \psi & \sin \psi \\
\sin \psi & \cos \psi
\end{array}\right]
$$

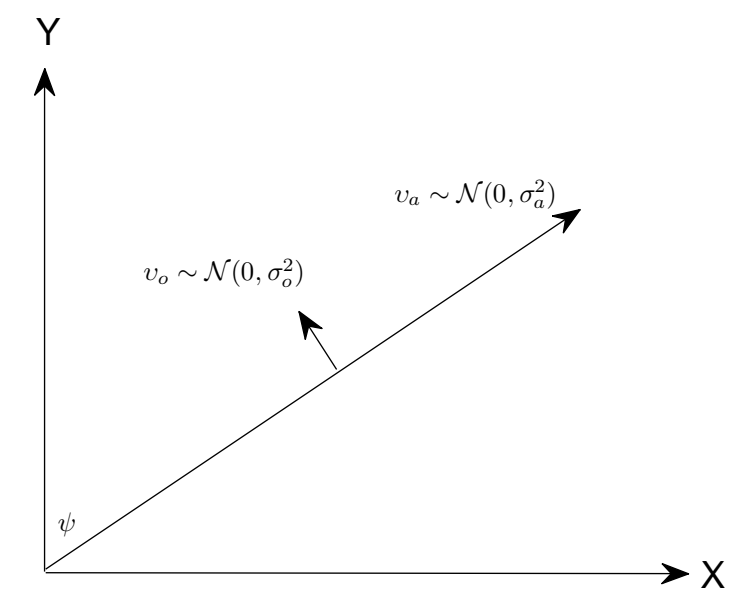

Figure 2.3: Directional process noise for path constrained targets 


\subsubsection{Velocity Prediction During Segment Changes}

Different segments may have different maximum velocities allowed based on terrain conditions. For example, maximum speed allowed in an urban area and rural area is not the same. Incorporating this maximum velocity constraint as a prior information in the prediction process can lead to a better estimate. Let target in a segment be $S_{k-1}$ where maximum velocity is $M V_{k-1}$ in time $(k-1)$ and the target is predicted to be in different segment $S_{k}$ with maximum velocity $M V_{k}$ in time $k$. If the resultant velocity of the target is $V(k-1 \mid k-1)$ in time $(k-1)$, the velocity in time $k$ is predicted to be $V(k \mid k-1)=\left(\frac{M V_{k}}{M V_{k-1}} V(k-1 \mid k-1)\right)$ in the $S_{k}$ segment direction. Here the assumption is target velocity change is proportional to the maximum velocity ratio and $V(k-1 \mid k-1)=\sqrt{\dot{x}(k-1 \mid k-1)^{2}+\dot{y}(k-1 \mid k-1)^{2}}$. Instead of this assumption, any other ratio that depends on the scenario can be integrated into proposed prediction/tracking algorithm as follows:

Let the ratio be $r$, then (2.10) is modified as

$$
R=\left[\begin{array}{cccc}
1 & 0 & 0 & 0 \\
0 & r \cos \theta & 0 & -r \sin \theta \\
0 & 0 & 1 & 0 \\
0 & r \sin \theta & 0 & r \cos \theta
\end{array}\right]
$$

Since the proposed algorithm explicitly considers the segment change at time $(k-\tau)$, velocity prediction is incorporated into the proposed algorithm in step 3 of Section 2. It can be noted here that it is not possible to include the above velocity ratio information in the existing algorithms (Kirubarajan et al., 2000), (Pannetier et al., 2005). 


\subsubsection{Hypothesis Generation Based on Segments}

A concept of hypotheses for segments (Pannetier et al., 2005) is used to handle the uncertainty in a junction. Assume a target moves in a segment $S_{k-1}$ at time $(k-1)$ and predicted state falls in more than one segment at time $k$, then for each segment a new segment hypothesis is generated. Now, the process of updating mode set and tracking are carried out for each segment hypothesis separately using a path constrained estimator. Consider a scenario where during the time interval $(k-3, k-2]$ target moves in segment $S_{1}$ and during the time interval $(k-2, k-1]$ target has the possibility of moving in either segment $S_{2}$ or $S_{3}$. Figure 2.4 shows segment hypothesis tree for the above scenario, where during time interval $(k-2, k-1]$ one segment hypothesis is available and during the next time interval two segment hypotheses are available.

Denote the hypotheses available during the time interval $(k-2, k-1]$ as $\Psi(k-1)=$ $\left(h_{1}^{\prime}(k-1), h_{2}^{\prime}(k-1) \ldots, h_{i}^{\prime}(k-1)\right)$. Similarly it can be written for the hypotheses available in time $(k-1, k]$ as $\Psi(k)$. The segment hypothesis probability is given by

$$
\begin{aligned}
\mu_{h}(k) & =P\left\{x(k) \in h \mid Z_{1}^{k}\right\} \\
& =\frac{1}{c} P\left[z(k) \mid Z_{1}^{(k-1)}, x(k) \in h\right] P\left[x(k) \in h \mid Z_{1}^{k-1}\right] \\
& =\frac{1}{c} \Delta_{h}(k) \sum_{h^{\prime} \in \Psi(k-1)} P\left[x(k) \in h \mid x(k) \in h^{\prime}, Z_{1}^{k-1}\right] P\left[x(k) \in h^{\prime} \mid Z_{1}^{k-1}\right] \\
& =\frac{1}{c} \Delta_{h}(k) \sum_{h^{\prime} \in \Psi(k-1)} p_{h^{\prime}, h} \mu_{h^{\prime}}(k-1)
\end{aligned}
$$

where $c$ is the normalization factor and $\Psi(k-1)$ is segment hypothesis transition probability. The probability of $\Delta_{h}(k)$ is shown in (Yeddanapudi et al., 1997) as 
follows:

$$
\begin{aligned}
\Delta_{h}(k) & =P\left\{Z_{1}^{k} \mid Z_{1}^{k-1}, x(k) \in h\right\} \quad \forall h \in \Psi(k) \\
& =\sum_{M \in h} \Lambda_{M}(k) \mu_{M}(k \mid k-1)
\end{aligned}
$$

where $M$ are the models belonging to the segments in segment hypothesis $h$ and $\mu_{M}(k \mid k-1)$ is the predicted mode probability.

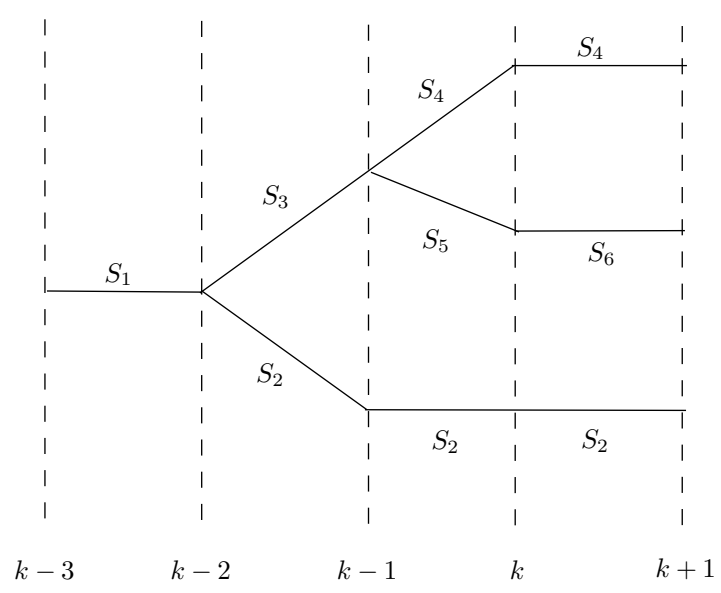

Figure 2.4: Segment hypothesis tree

Since more than one hypotheses are available at a time, there should be a mechanism to select the best hypotheses at a given time. A sequential probability ratio test as in (Pannetier et al., 2005) is carried out for this purpose. 


\section{Chapter 3}

\section{Retrodiction for Path Constrained}

\section{Targets}

Retrodiction is the estimation of the state at a time within the data time interval (Bar-Shalom et al., 2001). Since path information determines the smoothness of the track, no retrodiction algorithms have been specifically developed for path constrained problems in literature. A retrodiction algorithm for path constrained targets is developed in this research considering available path map information. An approach of a backward iteration scheme to calculate the states of the targets at a previous time given all measurement information is followed. The iteration process is initialized by the present target state and follows the standard RTS fixed interval smoothing (Rauch et al., 1965). In (Koch, 2000), fixed interval retrodiction to Multiple Hypothesis Tracking (MHT) applications employing IMM is given. The proposed algorithm here is an extended work of (Koch, 2000) with variable structure IMM for path constrained targets. While carrying out the tracking process, the mode set is updated at each segment change rather than at measurement receive time. This is considered 
in the following derivation during mode matched smoothing step. During a segment change in the prediction process, velocity is changed and predicted according to the segment constraint (Section 2.1.3). The velocity prediction is handled with the help of a rotation matrix (2.19) in prediction. Derived retrodiction algorithm considers the above modifications in retrodiction process, which results in better retrodicted states during the segment changes. A general approach is given for Gaussian smoothing in (Deisenroth and Ohlsson, 2011) and the derivation of the retrodiction algorithm here complies with this general approach.

Subsection 3.1 gives the steps for variable structure IMM retrodiction algorithm without segment constraint. This is similar to the algorithm proposed in (Nandakumaran et al., 2009) but differs in Step 3, mode matched retrodiction. Path constrained retrodiction algorithm is followed in subsection 3.2.

\subsection{General Retrodiction Algorithm}

Let $A(t)=\left(M_{1}(t), \ldots, M_{u}(t), \ldots\right)$ and $A(t+1)=\left(M_{1}(t+1), \ldots, M_{v}(t+1), \ldots\right)$. The models in $A(t)$ and $A(t+1)$ can be different and we are interested in $\hat{x}(t \mid k)$, where $t<k$. The steps for the retrodiction algorithm are

\section{Step 1 - Backward transition probability}

$$
\begin{aligned}
b_{v u} & =P\left(M_{u} \mid M_{v}, Z_{1}^{k}\right) \\
& =\frac{1}{d_{v}} p_{u v} \mu_{u}(t \mid t)
\end{aligned}
$$

where $d_{v}=\sum_{M_{u} \in A(t)} p_{u v} \mu_{u}(t \mid t)$ 


\section{Step 2 - Backward mixing probability}

$$
\begin{aligned}
\mu_{v \mid u}(t+1 \mid k) & =P\left(M_{v} \mid M_{u}, Z_{1}^{k}\right) \\
& =\frac{1}{d_{u}} b_{v u} \mu_{v}(t+1 \mid k)
\end{aligned}
$$

where $d_{u}=\sum_{M_{v} \in A(t+1)} b_{v u} \mu_{v}(t+1 \mid k)$

\section{Step 3 - Mode matched retrodiction}

$$
\begin{aligned}
\hat{x}^{u v}(t \mid k) & =\hat{x}^{u}(t \mid t)+A\left[\hat{x}^{v}(t+1 \mid k)-\hat{x}^{u v}(t+1 \mid t)\right] \\
P^{u v}(t \mid k) & =P^{u}(t \mid t)+A\left[P^{v}(t+1 \mid k)-P^{u v}(t+1 \mid t)\right] A^{T}
\end{aligned}
$$

where retrodiction gain $A$ is given by

$$
A=P^{u}(t \mid t)\left[F^{v}(t+1)\right]^{T}\left[F^{v}(t+1, t) P^{u}(t \mid t) F^{v}(t+1, t)^{T}+Q^{v}(t+1, t)\right]^{-1}
$$

Further the terms $\hat{x}^{u v}(t+1 \mid t)$ and $\hat{P}^{u v}(t+1 \mid t)$ are written as

$$
\begin{aligned}
\hat{x}^{u v}(t+1 \mid t) & =F^{v}(t+1, t) \hat{x}^{u}(t \mid t) \\
P^{u v}(t \mid k) & =F^{v}(t+1, t) P^{u}(t \mid t) F^{v}(t+1, t)^{T}+Q^{v}(t+1, t)
\end{aligned}
$$

During the retrodiction process, this iteration step will be initiated with present target state $\hat{x}^{v}(t+1 \mid k)=\hat{x}^{v}(k \mid k)$ 


\section{Step 4 - Mixing}

$$
\begin{aligned}
\hat{x}^{u}(t \mid k) & =\sum_{M_{v} \in A(t+1)} \hat{x}^{u v}(t \mid k) \mu_{v \mid u}(t+1 \mid k) \\
\hat{P}^{u}(t \mid k) & =\sum_{M_{v} \in A(t+1)} \mu_{v \mid u}(t+1 \mid k)\left(P^{u v}(t \mid k)+\left[\hat{x}^{u v}(t \mid k)-\hat{x}^{u}(t \mid k)\right]\left[\hat{x}^{u v}(t \mid k)-\hat{x}^{u}(t \mid k)\right]^{T}\right)
\end{aligned}
$$

\section{Step 5 - Smoothed mode Probability}

$$
\mu_{u}(t \mid k)=\frac{1}{f} \Lambda^{u}(t \mid k) \mu_{u}(t \mid t)
$$

where $f$ is a normalizing constant and

$$
\Lambda^{u}(t \mid k)=\sum_{M_{v} \in A(t+1)} p_{u v} \mathcal{N}\left(\hat{x}^{v}(t+1 \mid k) ; \hat{x}^{u v}(t+1 \mid t), P^{u v}(t+1 \mid t)\right)
$$

$$
\begin{aligned}
& \text { Step } 6 \text { - Smoothed Estimate } \\
& \begin{array}{l}
\hat{x}(t \mid k)=\sum_{M_{u} \in A(k)} \mu_{u}(t \mid k) \hat{x}^{u}(t \mid k) \\
\hat{P}(t \mid k)=\sum_{M_{u} \in A(k)} \mu_{u}(t \mid k)\left(\hat{P}^{u}(t \mid k)+\left[\hat{x}^{u}(t \mid k)-\hat{x}(t \mid k)\right]\left[\hat{x}^{u}(t \mid k)-\hat{x}(t \mid k)\right]^{T}\right)
\end{array}
\end{aligned}
$$

\subsection{Path Constrained Retrodiction Algorithm}

When there is a segment change between successively received measurement time, above retrodiction algorithm is modified to accommodate the segment changes and the corresponding motion model changes. The steps of the modified algorithm are: 
Let mode in effect during the time interval $(t, t+\delta]$ as $M_{u^{\prime}}$ and during $(t+\delta, t+1]$ as $M_{v^{\prime}}$ as shown in Figure 3.1, where $M_{u^{\prime}}, M_{v^{\prime}} \in A(t+1)$

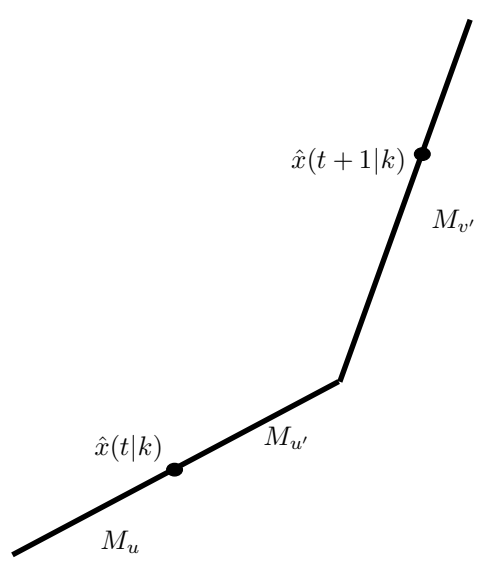

Figure 3.1: Mode changes at time $t+\delta$ due to segment change

\section{Step 1 - Backward transition probability}

$$
\begin{aligned}
b_{\left(v^{\prime} u^{\prime}\right) u} & =P\left(M_{u} \mid M_{\left(v^{\prime} u^{\prime}\right)}, Z_{1}^{k}\right) \\
& =\frac{1}{d_{\left(v^{\prime} u^{\prime}\right)}} p_{u^{\prime} v} p_{u u^{\prime}} \mu_{u}(t \mid t)
\end{aligned}
$$

where $d_{\left(v^{\prime} u^{\prime}\right)}=\sum_{M_{u} \in A(t)} p_{u^{\prime} v} p_{u u^{\prime}} \mu_{u}(t \mid t)$

\section{Step 2 - Backward mixing probability}

$$
\begin{aligned}
\mu_{\left(v^{\prime} u^{\prime}\right) \mid u}(t+1 \mid k) & =P\left(M_{v^{\prime}}, M_{u^{\prime}} \mid M_{u}, Z_{1}^{k}\right) \\
& =\frac{1}{d_{u}} b_{\left(v^{\prime} u^{\prime}\right) u} \mu_{\left(v^{\prime} u^{\prime}\right)}(t+1 \mid k)
\end{aligned}
$$

where $d_{u}=\sum_{M_{v^{\prime}}, M_{u^{\prime}} \in A(t)} b_{\left(v^{\prime} u^{\prime}\right) u} \mu_{\left(v^{\prime} u^{\prime}\right)}(t+1 \mid k)$ 


\section{Step 3 - Mode matched retrodiction}

$$
\begin{aligned}
& \hat{x}^{u\left(u^{\prime} v^{\prime}\right)}(t \mid k)=\hat{x}^{u}(t \mid t)+A\left[\hat{x}^{u^{\prime} v^{\prime}}(t+1 \mid k)-\hat{x}^{u\left(u^{\prime} v^{\prime}\right)}(t+1 \mid t)\right] \\
& P^{u\left(u^{\prime} v^{\prime}\right)}(t \mid k)=P^{u}(t \mid t)+A\left[P^{u^{\prime} v}(t+1 \mid k)-P^{u\left(u^{\prime} v^{\prime}\right)}(t+1 \mid t)\right] A^{T}
\end{aligned}
$$

where retrodiction gain $A$ is given by

$$
A=P^{u}(t \mid t)\left[F^{v^{\prime}}(t+1, t+\delta) R F^{u^{\prime}}(t+\delta, t)\right]^{T}\left[P^{u\left(u^{\prime} v^{\prime}\right)}(t+1 \mid t)\right]^{-1}
$$

Further the terms $\hat{x}^{u v}(t+1 \mid t)$ and $P^{u v}(t+1 \mid t)$ can be derived as

$$
\begin{aligned}
\hat{x}^{u\left(u^{\prime} v^{\prime}\right)}(t+1 \mid t) & =F^{v^{\prime}}(t+1, t+\delta) R F^{u^{\prime}}(t+\delta, t) \hat{x}^{u}(t \mid t) \\
P^{u\left(u^{\prime} v^{\prime}\right)}(t+1 \mid t) & =F^{v^{\prime}}(t+1, t+\delta) R\left\{F^{u^{\prime}}(t+\delta, t) P^{u}(t \mid t) F^{u^{\prime}}(t+\delta, t)^{T}\right. \\
& \left.+Q^{u^{\prime}}(t+\delta, t)\right\} R^{T} F^{v^{\prime}}(t+1, t+\delta)^{T}+Q^{v^{\prime}}(t+1, t+\delta)
\end{aligned}
$$

Here all terms except $\hat{x}^{u^{\prime} v^{\prime}}(t+1 \mid k)$ and $P^{u^{\prime} v}(t+1 \mid k)$ can be calculated and stored during the prediction process to be used for retrodiction.

\section{Step 4 - Mixing}

$$
\begin{aligned}
\hat{x}^{u}(t \mid k) & =\sum_{M_{v^{\prime}}, M_{u^{\prime}} \in A(t+1)} \hat{x}^{u\left(u^{\prime} v^{\prime}\right)}(t \mid k) \mu_{\left(v^{\prime} u^{\prime}\right) \mid u}(t+1 \mid k) \\
\hat{P}^{u}(t \mid k) & =\sum_{M_{v^{\prime}}, M_{u^{\prime}} \in A(t+1)} \mu_{\left(v^{\prime} u^{\prime}\right) \mid u}(t+1 \mid k)\left(P^{u\left(u^{\prime} v^{\prime}\right)}(t \mid k)\right. \\
& \left.+\left[\hat{x}^{u\left(u^{\prime} v^{\prime}\right)}(t \mid k)-\hat{x}^{u}(t \mid k)\right]\left[\hat{x}^{u\left(u^{\prime} v^{\prime}\right)}(t \mid k)-\hat{x}^{u}(t \mid k)\right]^{T}\right)
\end{aligned}
$$




\section{Step 5 - Smoothed mode Probability}

$$
\mu_{u}(t \mid k)=\frac{1}{f} \Lambda^{u}(t \mid k) \mu_{u}(t \mid t)
$$

where $f$ is a normalizing constant and

$$
\Lambda^{u}(t \mid k)=\sum_{M_{v^{\prime}}, M_{u^{\prime}} \in A(t+1)} p_{u^{\prime} v^{\prime}} p_{u u^{\prime}} \mathcal{N}\left(\hat{x}^{u^{\prime} v^{\prime}}(t+1 \mid k) ; \hat{x}^{u\left(u^{\prime} v^{\prime}\right)}(t+1 \mid t), P^{u\left(u^{\prime} v^{\prime}\right)}(t+1 \mid t)\right)
$$

Step 6 - Smoothed Estimate

$$
\begin{aligned}
& \hat{x}(t \mid k)=\sum_{M_{u} \in A(k)} \mu_{u}(t \mid k) \hat{x}^{u}(t \mid k) \\
& \hat{P}(t \mid k)=\sum_{M_{u} \in A(k)} \mu_{u}(t \mid k)\left(\hat{P}^{u}(t \mid k)+\left[\hat{x}^{u}(t \mid k)-\hat{x}(t \mid k)\right]\left[\hat{x}^{u}(t \mid k)-\hat{x}(t \mid k)\right]^{T}\right)
\end{aligned}
$$

\subsection{Posterior Cramer Rao Lower Bound for Path Constrained Targets}

Posterior Cramer Rao Lower Bound (PCRLB) gives a lower bound on the error covariance and it is defined by inverse, $J(k)^{-1}$ of the Fisher information matrix (FIM)

$$
\mathbf{E}\left[[\hat{x}(k)-x(k)][\hat{x}(k)-x(k)]^{T}\right] \geq J(k)^{-1}
$$

where $\mathbf{E}$ denotes expectation over $\left(x(k), Z_{1}^{k}\right)$.

For a linear system without measurement origin uncertainty, $J(k+1)$ can be 
written as sum of (Hernandez et al., 2004)

$$
J(k+1)=\left[Q(k)+F(k) J(k)^{-1} F(k)^{T}\right]^{-1}+\sum_{i=1}^{n} \mathbf{E}\left(H^{i}(k) \Sigma^{i}(k)^{-1} H^{i}(k)^{T}\right)
$$

where $n$ is the number of sensors.

However, it has been observed that the PCRLB can be overly optimistic in certain cases (Horridge and Hernandez, 2003), (Hernandez, 2003) and (Hernandez et al., 2008). In (Hernandez, 2003) it is shown that PCRLB for tracking road-based vehicles using ground moving target indicator (GMTI) sensors were over-optimistic, due to the potential multi-modality of the target distribution at each junction. It is shown in (Hernandez et al., 2008) that PCRLB bound for tracking manoeuvering target with Markovian switching dynamics produce an overly optimistic lower bound due to the assumption of implicitly assumed known manoeuvres. As a result instead of PCRLB, a performance measure is given for Markovian switching dynamic systems in (Hernandez et al., 2008). So to evaluate the proposed path constrained algorithm with PCRLB, a single model is considered for developing the bound for path constrained targets instead of multi-model Markovian switching dynamics.

The challenging part in path constrained tracking PCRLB is to consider the structure of the road network and the constraints imposed on the target motion. In (Hernandez, 2003), when a target reaches a junction all possible path available at the junction are incorporated for the PCRLB calculation, which resulted in over optimistic PCRLB. But the path prediction algorithm developed in this paper considers sequence of path as different segment hypotheses as described in subsection 2.1.4. This allows PCRLB to be derived for each segment hypothesis separately and based 
on the technique described in (Hernandez, 2003), PCRLB for path constrained targets is developed with single model tracking.

Consider Figure 2.1, and let the time duration between successive scan $k$ and $(k+1)$ be $T$, and time taken to reach the intersection point is $T 1$. Then the equivalent state transition matrix, $F_{e q}$, required for the PCRLB calculation when there is a segment change in target motion is given by

$$
F_{e q}=F(T-T 1) R F(T 1)
$$

where $F(T 1)$ is the state transition matrix correspond to a motion model for time duration $T 1$ in segment $S_{k-1}, F(T-T 1)$ correspond to a motion model for time duration (T-T1) in segment $S_{k}$ and $R$ is the rotation matrix given in (2.10). Process noise covariance $Q(k)$ is replaced with directional process noise covariance in (3.28) and when there is a segment change in the target motion it can be shown that the equivalent process noise covariance, $Q_{e q}$, is given by

$$
Q_{e q}=F(T-T 1) R Q(T 1) R^{T} F(T-T 1)^{T}+Q(T-T 1)
$$

where $Q(T 1)$ is the directional process noise for time duration $T 1$ and $Q(T-T 1)$ is the directional process noise for time duration $(T-T 1)$.

Further PCRLB for retrodiction without measurement origin uncertainty is same as RTS equation (Simandl et al., 2001). Retrodiction algorithm developed in this paper for path constrained targets follows the concept of RTS equation and it can be shown that for a single model retrodiction PCRLB for path constrained targets can be derived from single model version of (3.16) and (3.17). 


\section{Chapter 4}

\section{Simulation Studies and Results}

To validate the proposed algorithms, simulations are carried out and analyzed. The simulated scenario based test is given in section 4.1 and a test on the real data gathered from exactEarth Inc is given in section 4.2.

\subsection{Simulation Test}

The path map network used to demonstrate the simulation is shown in Figure 4.1. There are five segments and six way-points with two junctions. For simulation purposes it is assumed that probability of a target moving to segment $S_{2}$ from $S_{1}$ is 0.2 and probability of a target moving to segment $S_{3}$ from $S_{1}$ is 0.8 . Similarly probability for $S_{3}$ to $S_{4}$ is 0.2 and $S_{3}$ to $S_{5}$ is 0.8 . With this segment transition probabilities target trajectories are generated for 100 Monto carlo runs. Target's dynamic variation is given for segment sequence $\left(S_{1}, S_{3}, S_{5}\right)$ in Figure 4.2 where target reaches the intersection points at approximately $510 \mathrm{~s}$ and $1650 \mathrm{~s}$. Target state $\mathbf{x}(k)$ is defined by the 4 -dimensional vector $\mathbf{x}(k)=\left[\begin{array}{llll}x(k) & \dot{x}(k) & y(k) & \dot{y}(k)\end{array}\right]^{T}$ where $x(k)$ and $\dot{x}(k)$ are 
position and velocity of a target in $X$ direction. The sensor measurement consists of target's position in $X$ and $Y$ direction resulting in observation matrix

$$
H(k)=\left[\begin{array}{llll}
1 & 0 & 0 & 0 \\
0 & 0 & 1 & 0
\end{array}\right]
$$

Second order white noise acceleration models are used for modeling the motion of target, where second order piecewise white noise acceleration model state transition matrix $F(k, k-1)$ is given by

$$
F(k, k-1)=\left[\begin{array}{cccc}
1 & T & 0 & 0 \\
0 & 1 & 0 & 0 \\
0 & 0 & 1 & T \\
0 & 0 & 0 & 1
\end{array}\right]
$$

and vector gain $\Gamma(k, k-1)$ is given by

$$
\Gamma(k, k-1)=\left[\begin{array}{cc}
T^{2} / 2 & 0 \\
T & 0 \\
0 & T^{2} / 2 \\
0 & T
\end{array}\right]
$$

here $T$ is the time duration between scan $(k-1)$ and $k$. The fixed mode IMM estimator, which is used for comparing simulation results with proposed algorithm, consists of two second order white noise acceleration models with equal process noise levels in both the $X$ and $Y$ directions. For these filters, $\sigma_{x}=\sigma_{y}$ were 0.05 and 0.5 $\mathrm{m} / \mathrm{s}^{2}$, which corresponds to constant velocity and maneuver modes, respectively. 
The proposed path constrained filter used two models for the path constrained motion. Constrained constant velocity model defined by $\sigma_{a}=0.05 \mathrm{~m} / \mathrm{s}^{2}$ and $\sigma_{o}=0.002$ $\mathrm{m} / \mathrm{s}^{2}$, and a manoeuvre model defined by $\sigma_{a}=0.5 \mathrm{~m} / \mathrm{s}^{2}$ and $\sigma_{o}=0.002 \mathrm{~m} / \mathrm{s}^{2}$. Sensor revisit interval is 60 seconds with error covariance of $400 \mathrm{~m}^{2}$ in both $X$ and $Y$ position. A single target with zero process noise is considered with the detection probability equals to unity and false alarm probability equals to zero. The sojourn time (Bar-Shalom et al., 2001) for the above models are 100s. The benefits of using the proposed algorithm are quantified using the results from 100 Monte Carlo runs.

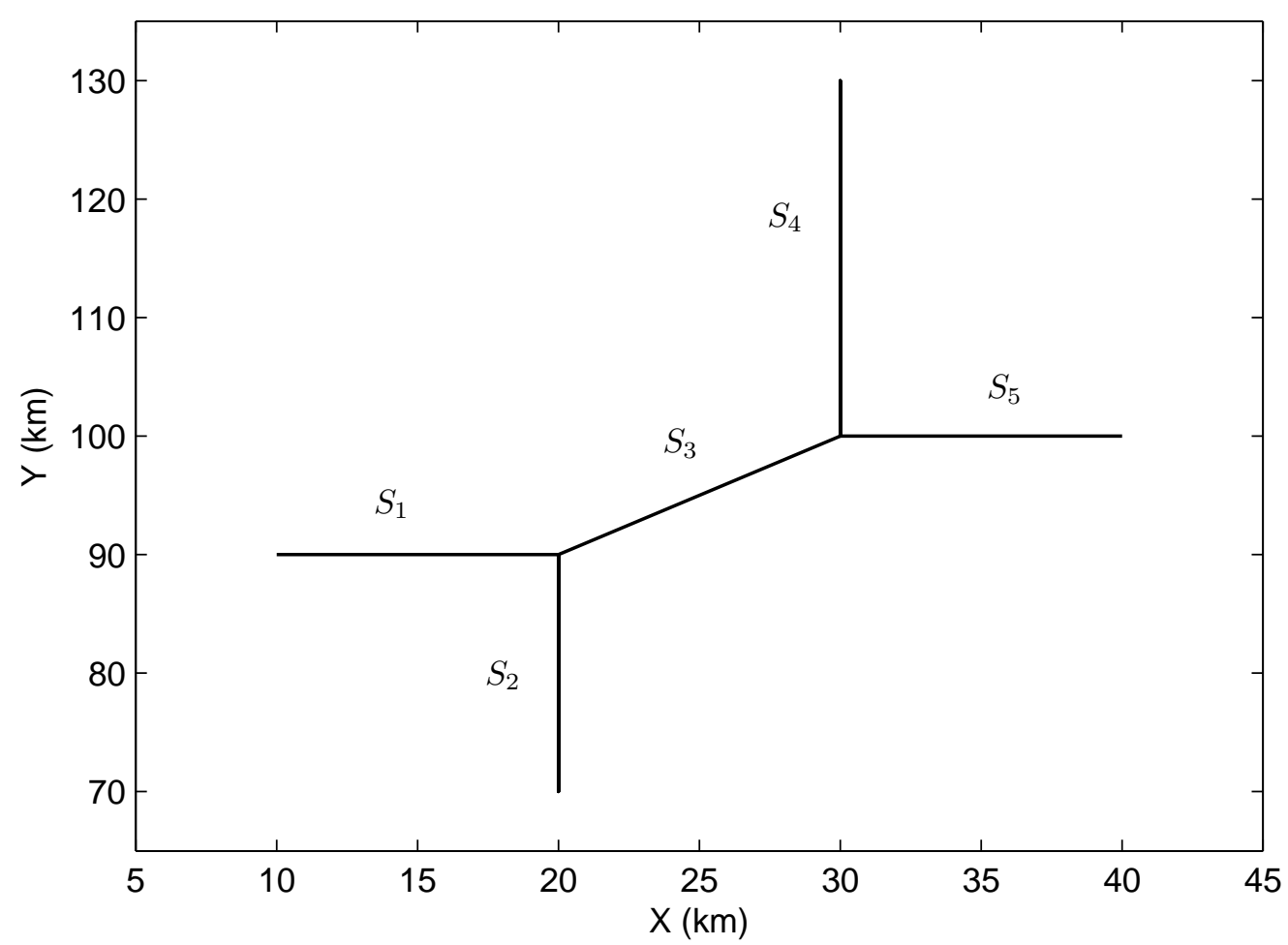

Figure 4.1: Path map for simulation 


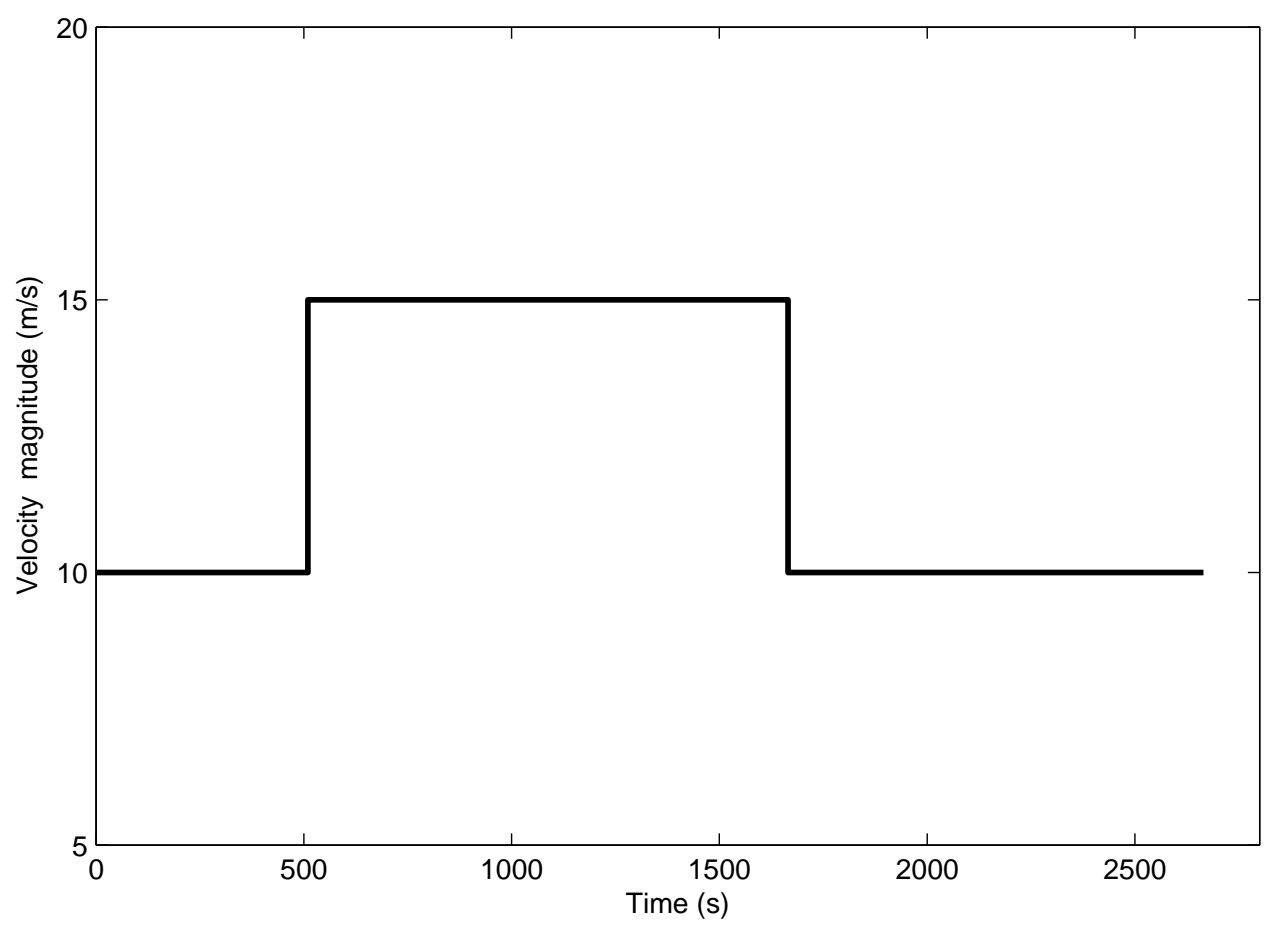

Figure 4.2: Target dynamics variation

There are three segment hypotheses possible for this scenario. The segment sequences of these segment hypotheses are $\left(S_{1}, S_{2}\right),\left(S_{1}, S_{3}, S_{4}\right)$ and $\left(S_{1}, S_{3}, S_{5}\right)$. But when the measurements become available, hypotheses will be pruned based on the sequential probability ratio test. The simulation results given here are based on the best hypothesis selected from each Monte Carlo run. Figure 4.3 shows the Root Mean Square Error (RMSE) in position for tracking. Proposed algorithm is compared against fixed mode IMM, variable structure IMM and measurements. Variable structure IMM used the same motion models as proposed path constrained estimator. Proposed path constrained algorithm performs better than the other algorithms due to the better prediction mechanism, where additional prior information of path map 
and imposed constraints are used. For instance, predicted state position was close to the segment $S_{3}$ in segment sequence $\left(S_{1}, S_{3}, S_{4}\right)$ when the target was approaching the intersection between segment $S_{1}$ and $S_{3}$ where IMM prediction mechanism will predict it in an off-path position. Since same motion models are used for path constrained algorithm and VS-IMM, position RMSE is same except the region near the junctions. When a target is within the validation gate (Kirubarajan et al., 2000) of a junction, all the motion models corresponding to all possible segments associated to the junction are added to the mode set in VS-IMM. For e.g., when a target is within the validation gate of the first junction, all motion models corresponding to segments $S_{1}, S_{2}$ and $S_{3}$ are added. The problem with this method is if the target is following segment sequence $\left(S_{1}, S_{3}, S_{4}\right)$, adding the motion models corresponding to segment $S_{2}$ is increasing the position error and position uncertainty. Because of the models which are actually not relevant to the target motion also added, the position error becomes larger near the junctions for VS-IMM. But the proposed algorithm generates segment hypotheses (2.1.4) and modes are added based on the segments involved in a segment hypothesis. For e.g., when the target is closer to the first junction two hypotheses with segment sequences $\left(S_{1}, S_{2}\right)$ and $\left(S_{1}, S_{3}\right)$ will be generated and mode set will be updated for each segment hypothesis. This segment hypothesis approach with prior information of maximum velocity for a segment, results in better performance in position RMSE (Figure 4.3) for path constrained algorithm over VS-IMM near the junctions. Further it can be seen (Figure 4.3) that fixed mode IMM performs slightly better than the measurements for the above scenario with given fixed mode models. But when the process noise of the fixed mode IMM models are increased the performance of fixed mode IMM degrades as expected. 
Figure 4.4 shows the Root Mean Square Error (RMSE) in velocity for tracking. The improvement in velocity RMSE is due to the velocity rotation, maximum velocity ratio and segment hypothesis approach, which are considered during the prediction. For example, when a target moves from $S_{1}$ to $S_{3}$, direction of the velocity changes by 30 degrees and velocity is increased by 1.5 times. These changes are captured by the algorithm in the introduced rotation matrix but not in the standard IMM structure or VS-IMM, which results in more visible difference between algorithms. The better performance of the path constrained algorithm and VS-IMM over fixed mode IMM during the other time intervals is due to the directional process noise models used in VS-IMM and proposed algorithm.

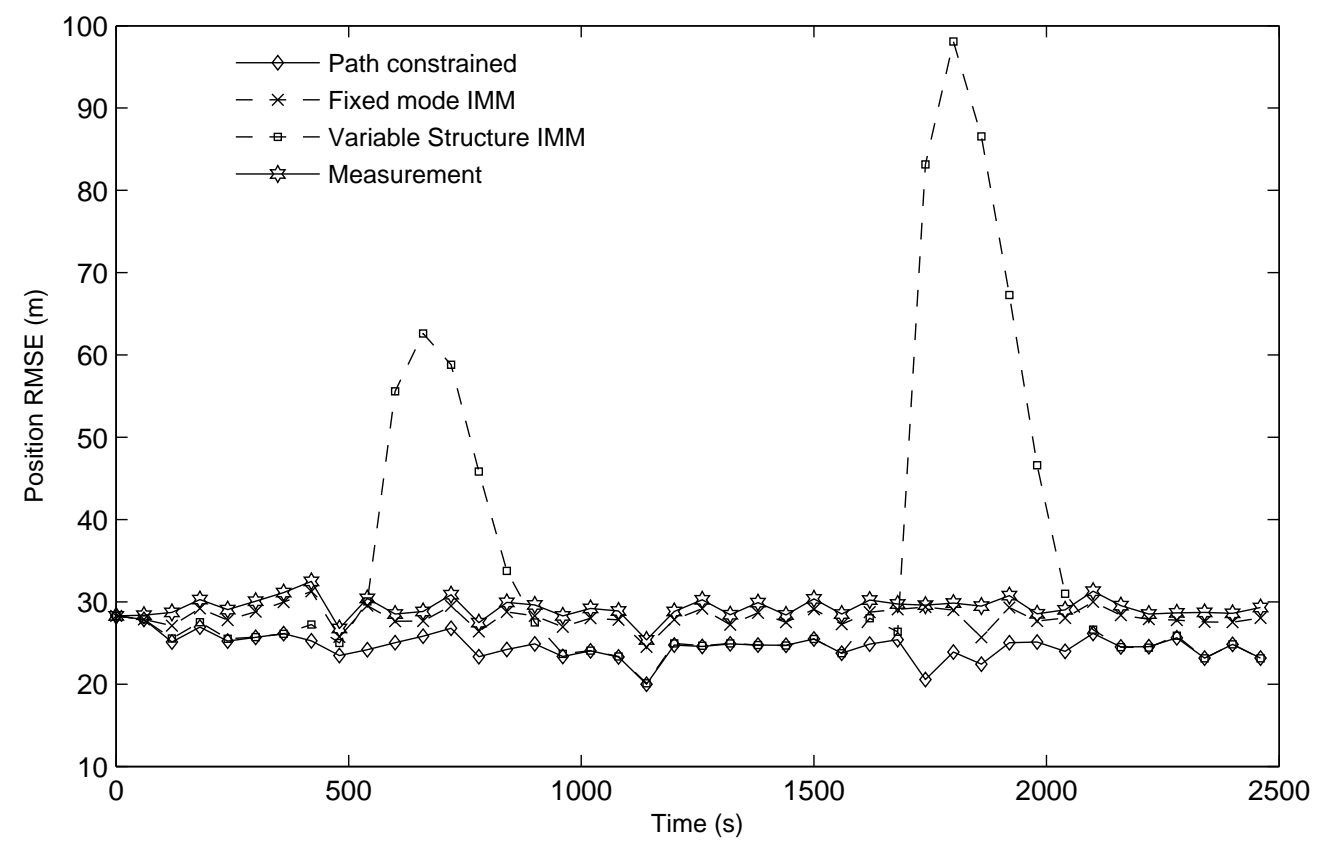

Figure 4.3: RMS position errors for estimators 


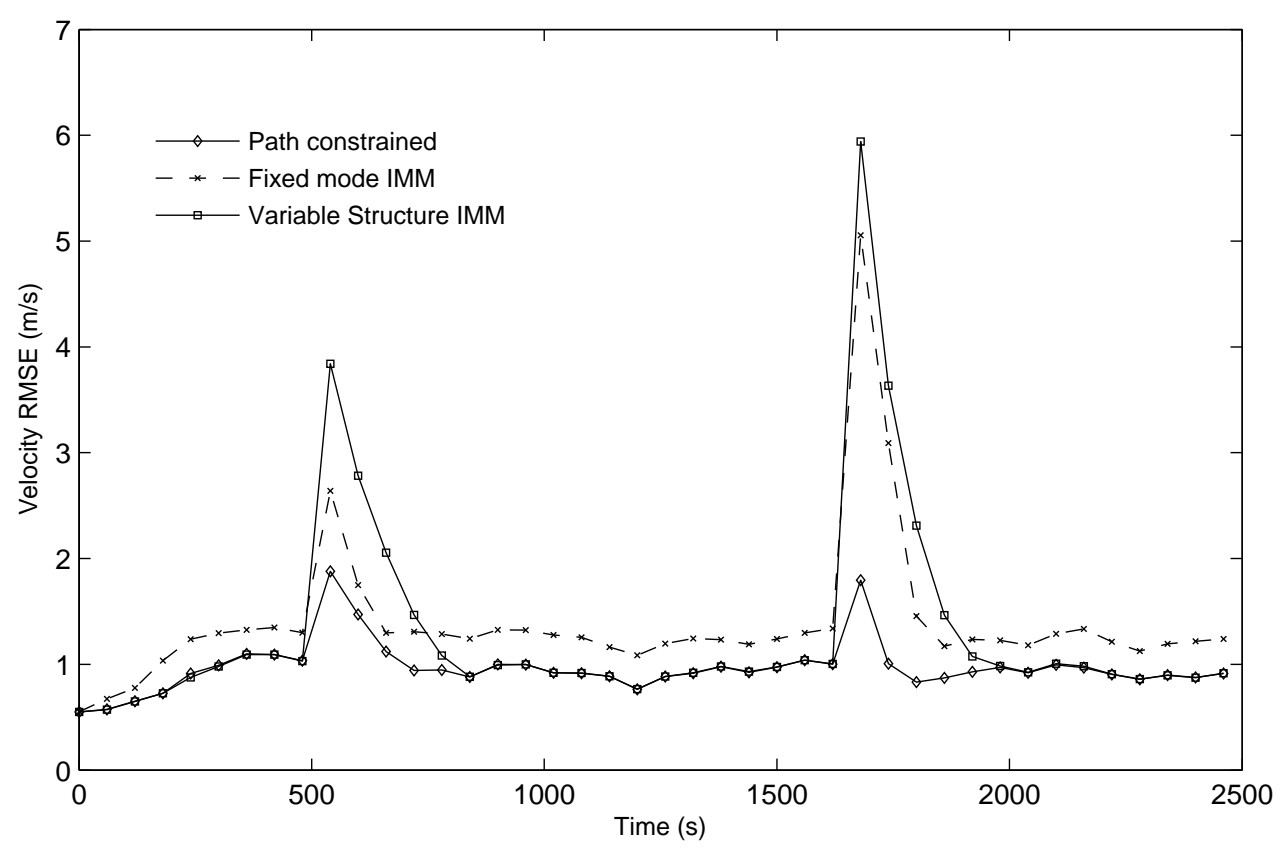

Figure 4.4: RMS velocity errors for estimators

Figure 4.5 and 4.6 shows root mean square error (RMSE) in position and velocity respectively for retrodiction compared with fixed interval IMM retrodiction algorithm (Koch, 2000). It is clearly seen that the IMM retrodiction algorithm shows a degraded performance in the junction compared to the proposed retrodiction algorithm. It is due to the predicted state (3.19) and retrodiction gain (3.18) in path constrained retrodiction algorithm are calculated considering the segment change and motion model change in the junctions where IMM retrodiction algorithm does not. As a result, a spike is observed during the intersection points. It has to be noted that the predicted state (3.19) and retrodiction gain (3.18) can be calculated and stored during the prediction stage to reduce the computational load during retrodiction process though it increases the memory load. During the other time intervals, due 
to the advantage of directional process noise concept better performance of proposed retrodiction algorithm is observed over the fixed interval IMM retrodiction algorithm, which used the estimates from fixed mode IMM.

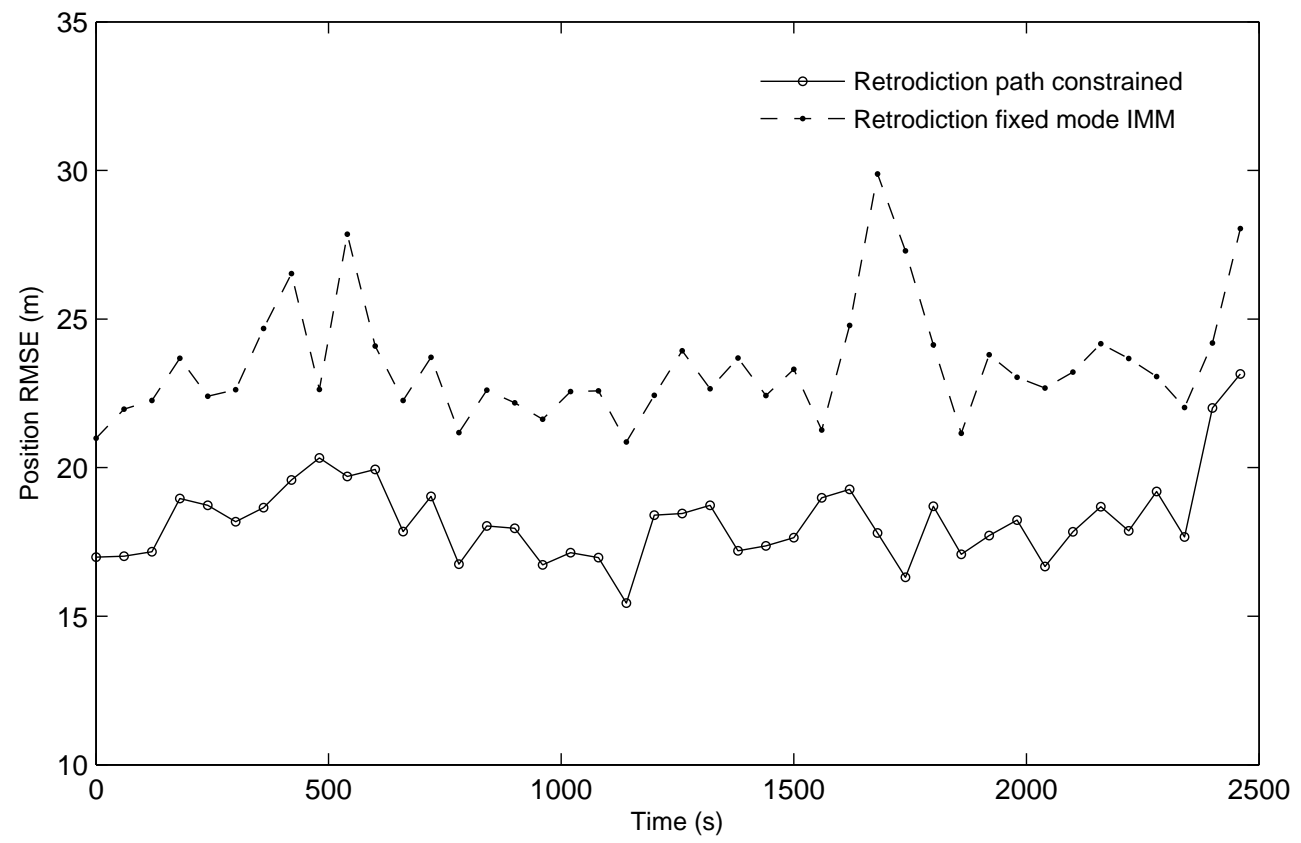

Figure 4.5: RMS position errors for retrodiction 


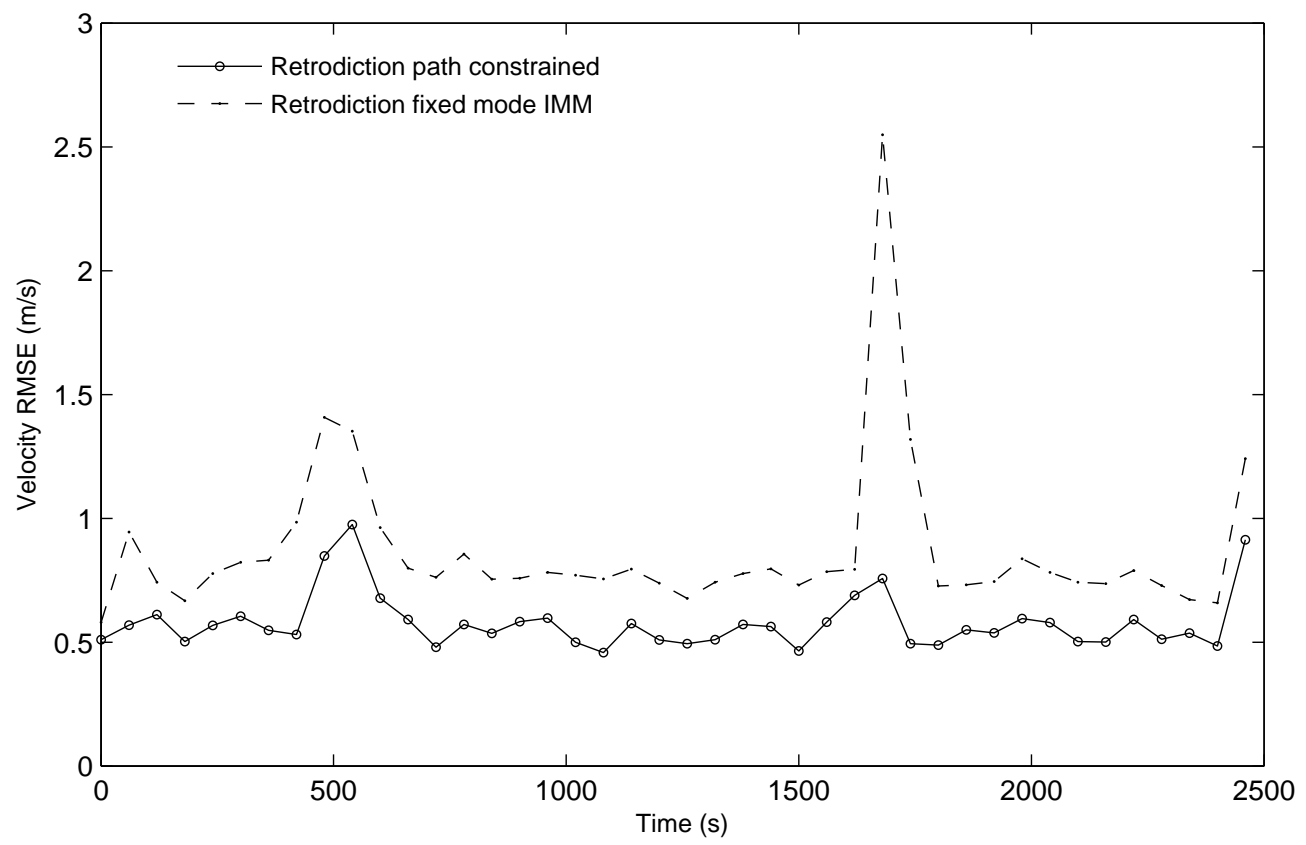

Figure 4.6: RMS velocity errors for retrodiction

Figure 4.7 and Figure 4.8 shows the comparison between the proposed tracking and proposed retrodiction algorithms where it can be clearly seen the improvement of retrodiction algorithm compared to the tracking algorithm over the whole time period. 


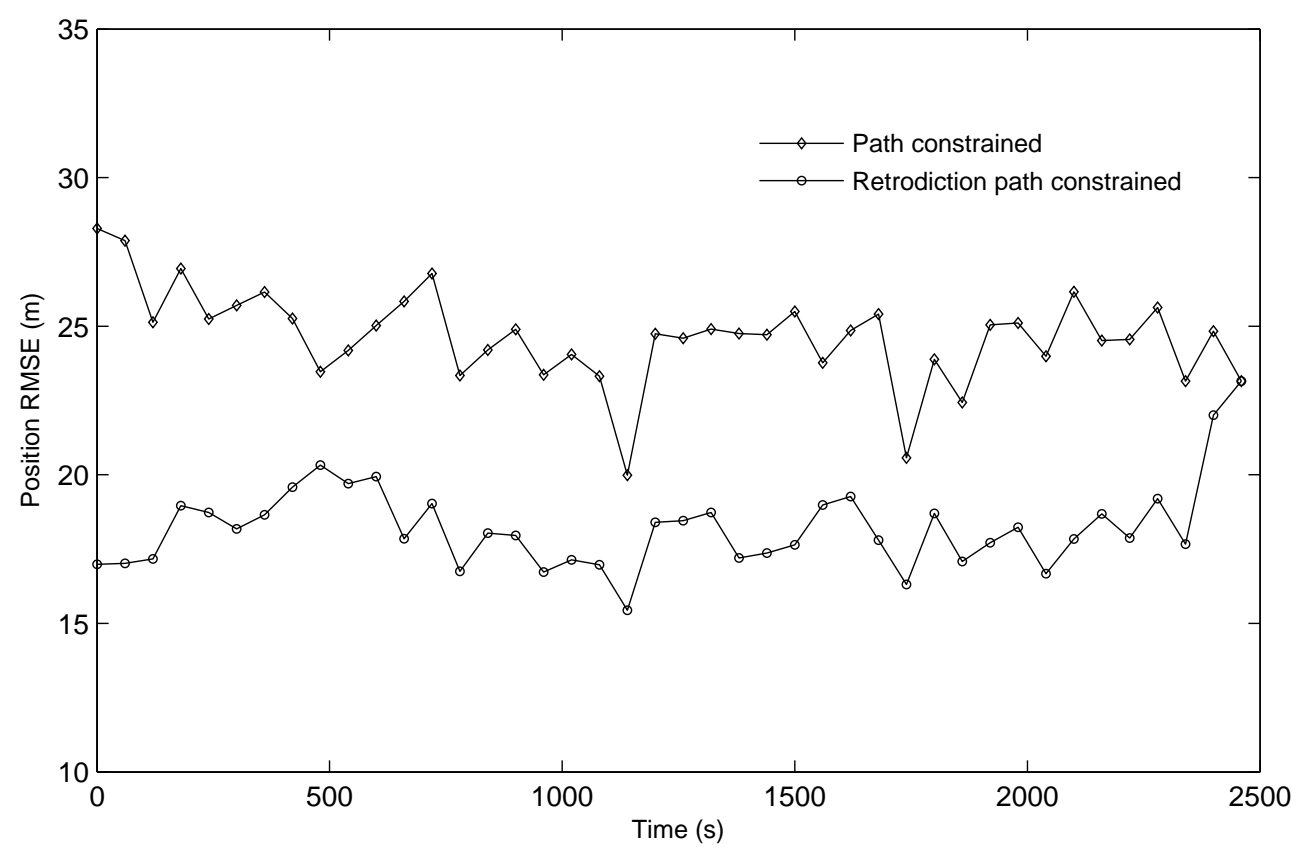

Figure 4.7: RMS position errors comparison for proposed algorithms 


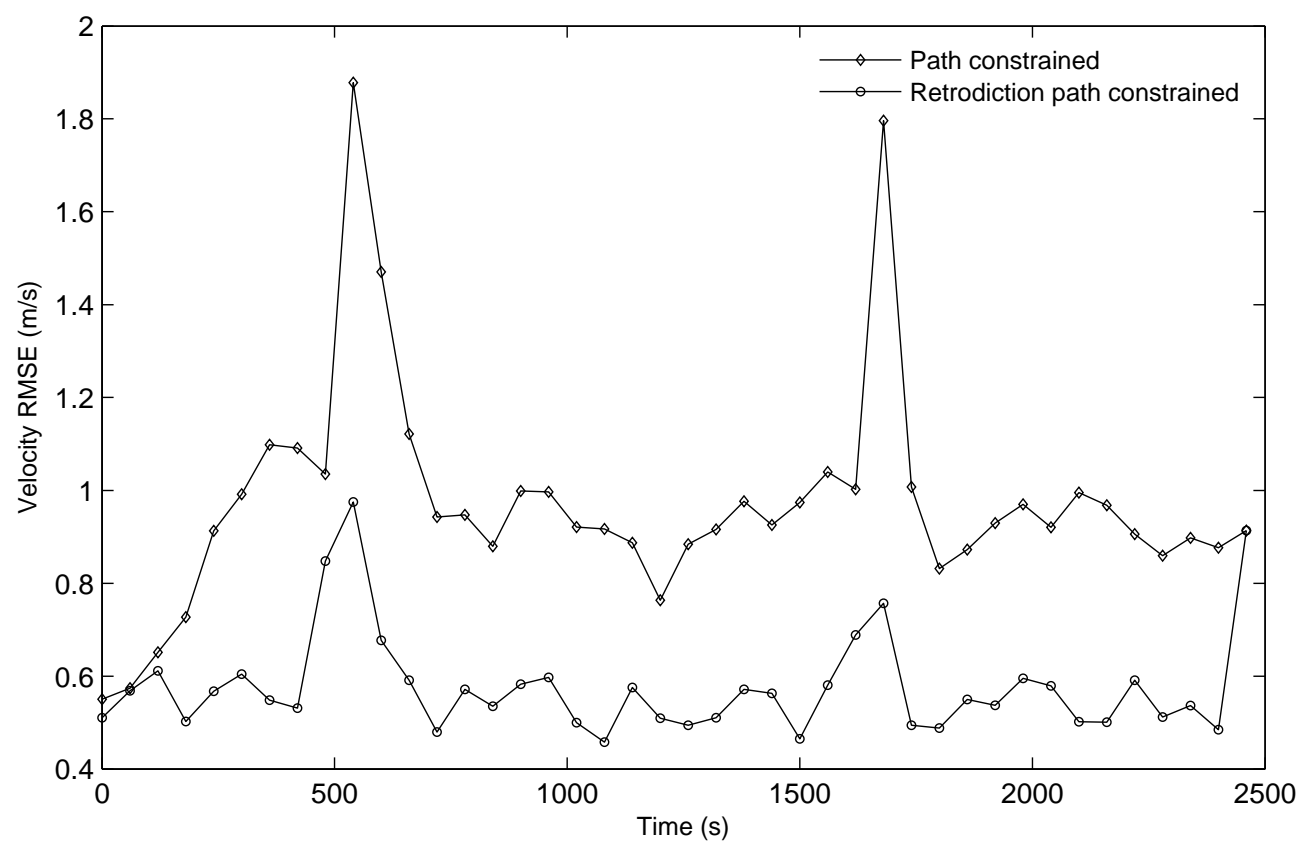

Figure 4.8: RMS velocity errors comparison for proposed algorithms 
Figure 4.9 shows the PCRLB bound for the tracking algorithm compared to the proposed path constrained algorithm's RMSE. PCRLB is calculated here with a single constant velocity motion model and the proposed tracking algorithm RMSE lies within the bound of PCRLB. Table 4.1 shows the average performance metrics in summary for the above simulations carried out. Based on these observations, one can rank the algorithms as follows(in order of increasing performance):

1. Fixed mode IMM

2. Path constrained filter

3. Retrodiction algorithm IMM-MHT

4. Retrodiction algorithm path constrained filter

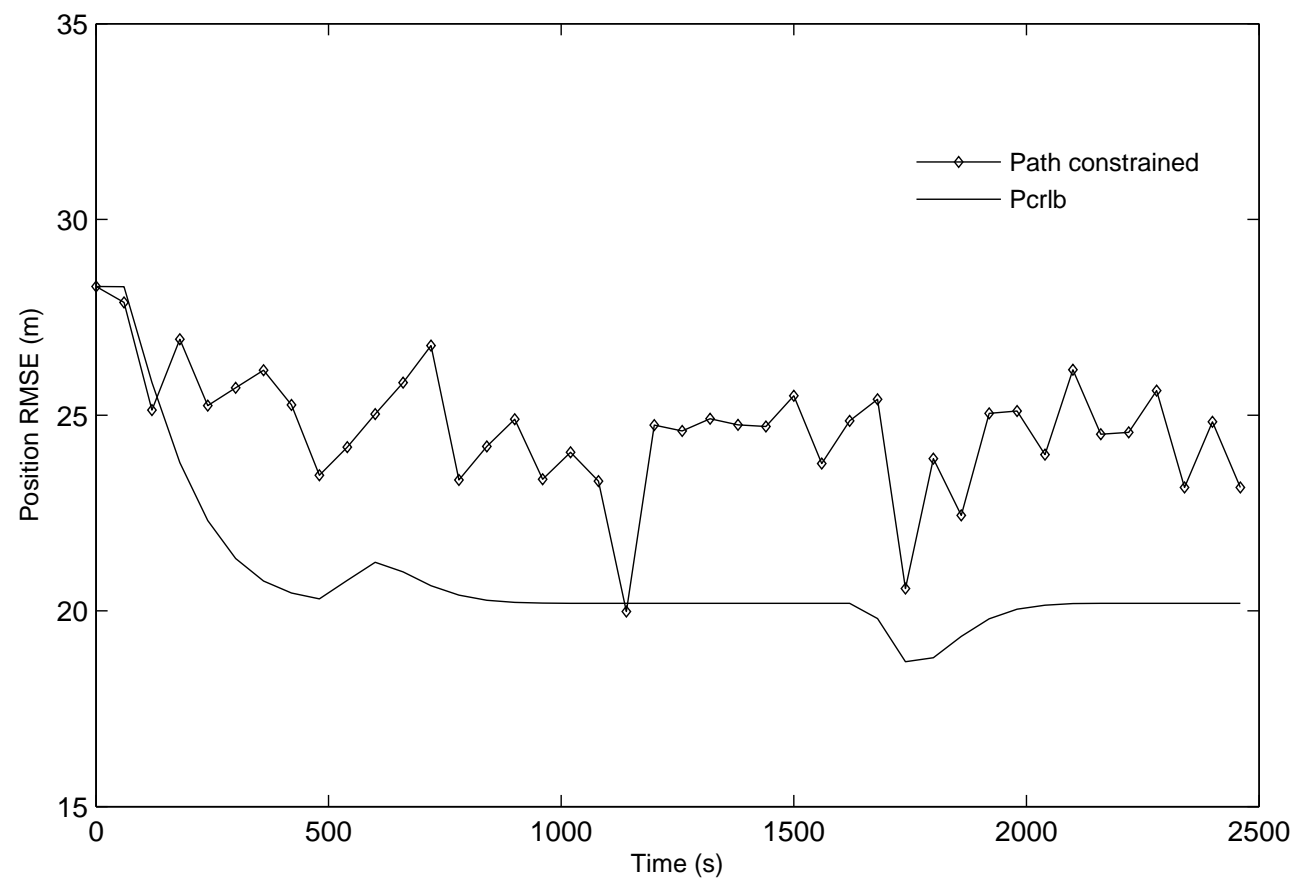

Figure 4.9: PCRLB position comparison

Further, the computational load of the proposed path constrained algorithm is $2 \%$ 
Table 4.1: Average performance metrics

\begin{tabular}{|l|l|l|}
\hline Filter & RMS pos. error $(\mathrm{m})$ & RMS vel. error(m/s) \\
\hline Fixed mode IMM & 27.85 & 1.56 \\
\hline Path constrained filter & 24.78 & 1.02 \\
\hline Retrodiction IMM-MHT & 23.46 & 0.93 \\
\hline Retrodiction path constrained filter & 18.98 & 0.60 \\
\hline
\end{tabular}

higher than the VS-IMM algorithm and proposed retrodiction algorithm is $5 \%$ higher than the fixed mode retrodiction IMM algorithm.

\subsection{Real Data Test}

Satellite-based AIS data is used for this test. This data corresponds to the vessels in region close to Indonesia and collected during September, 2011. Figure 4.10 shows the location (from 'Google Maps') corresponding to the above region. Since it is a path constrained problem, a path map should be available as a priori information. Due to the unavailability of shipping lanes in the corresponding region, latitude and longitude measurements from the given data set file is used to generate the path map. Figure 4.11 shows the whole measurement set plotted and this figure is used to generate the path map. But Matlab can not handle thousands of targets with the required plotting facility for prediction and retrodiction, due to that a specific area is selected for simulation as shown in the Figure 4.11.

Figure 4.12 shows the path map developed manually with 8 way-points and 7 segments for this test. Initially around 25 targets close to the selected area are taken to predict and track. Then two targets are selected to run and get the RMS position errors. 


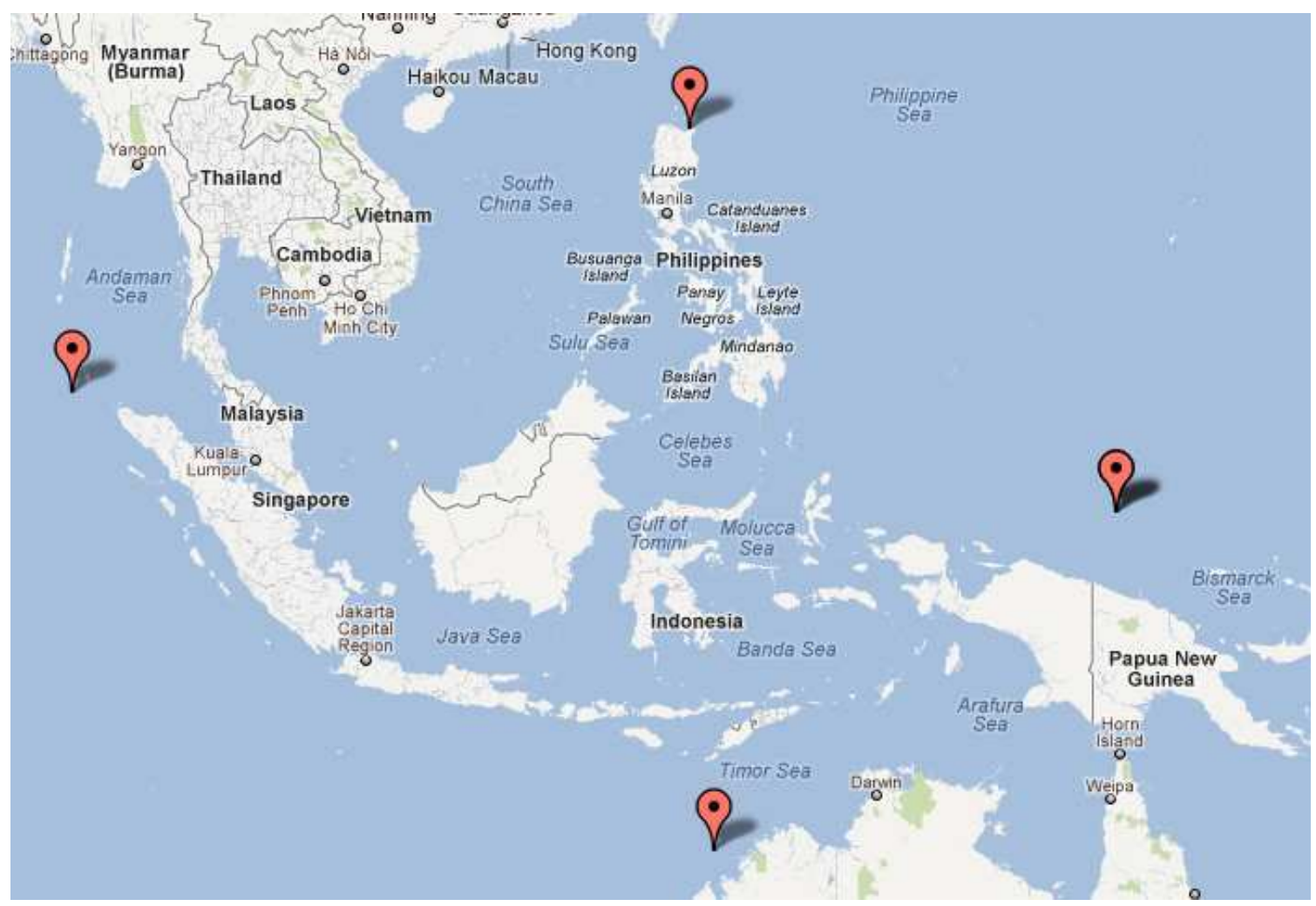

Figure 4.10: Location (Google maps) 


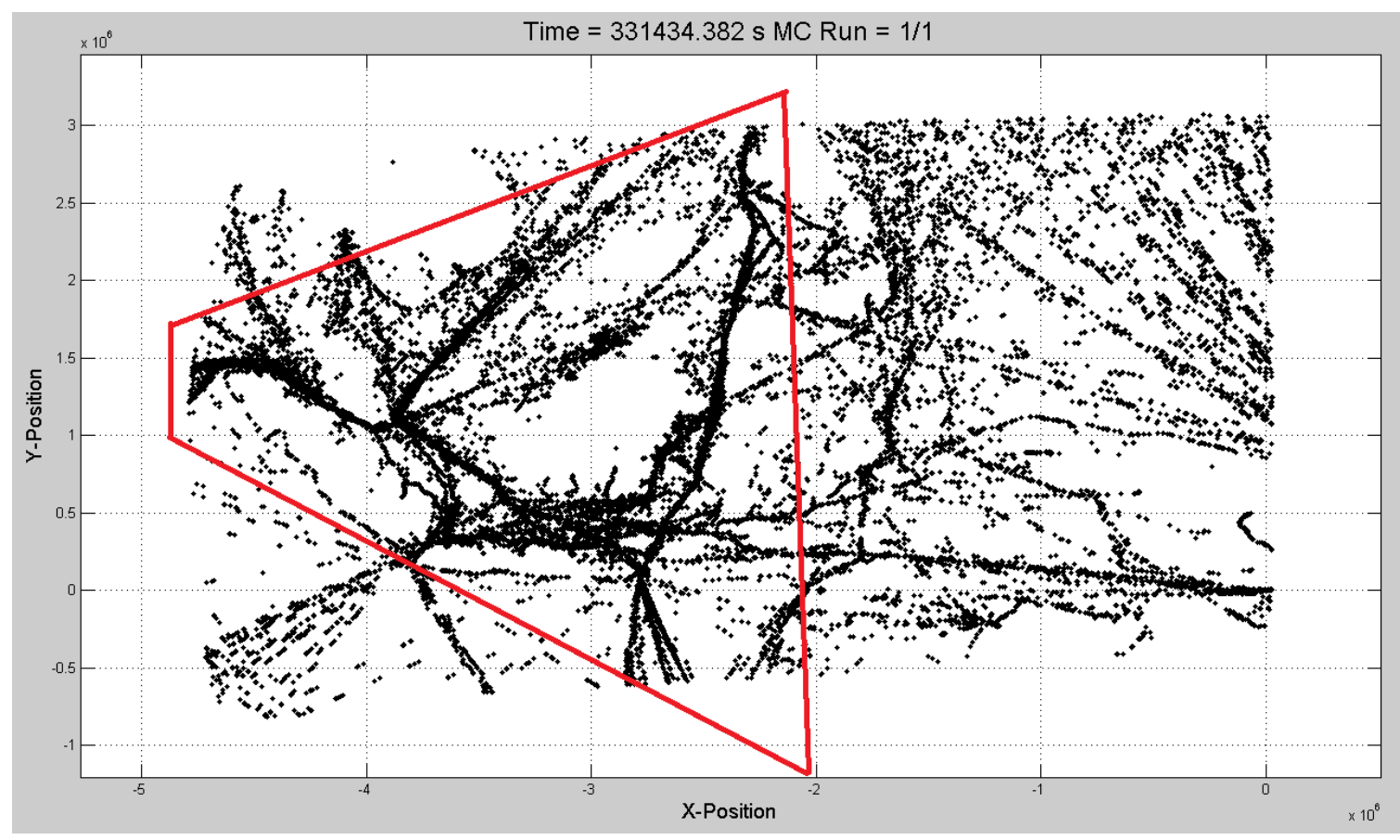

Figure 4.11: Whole measurements plot 


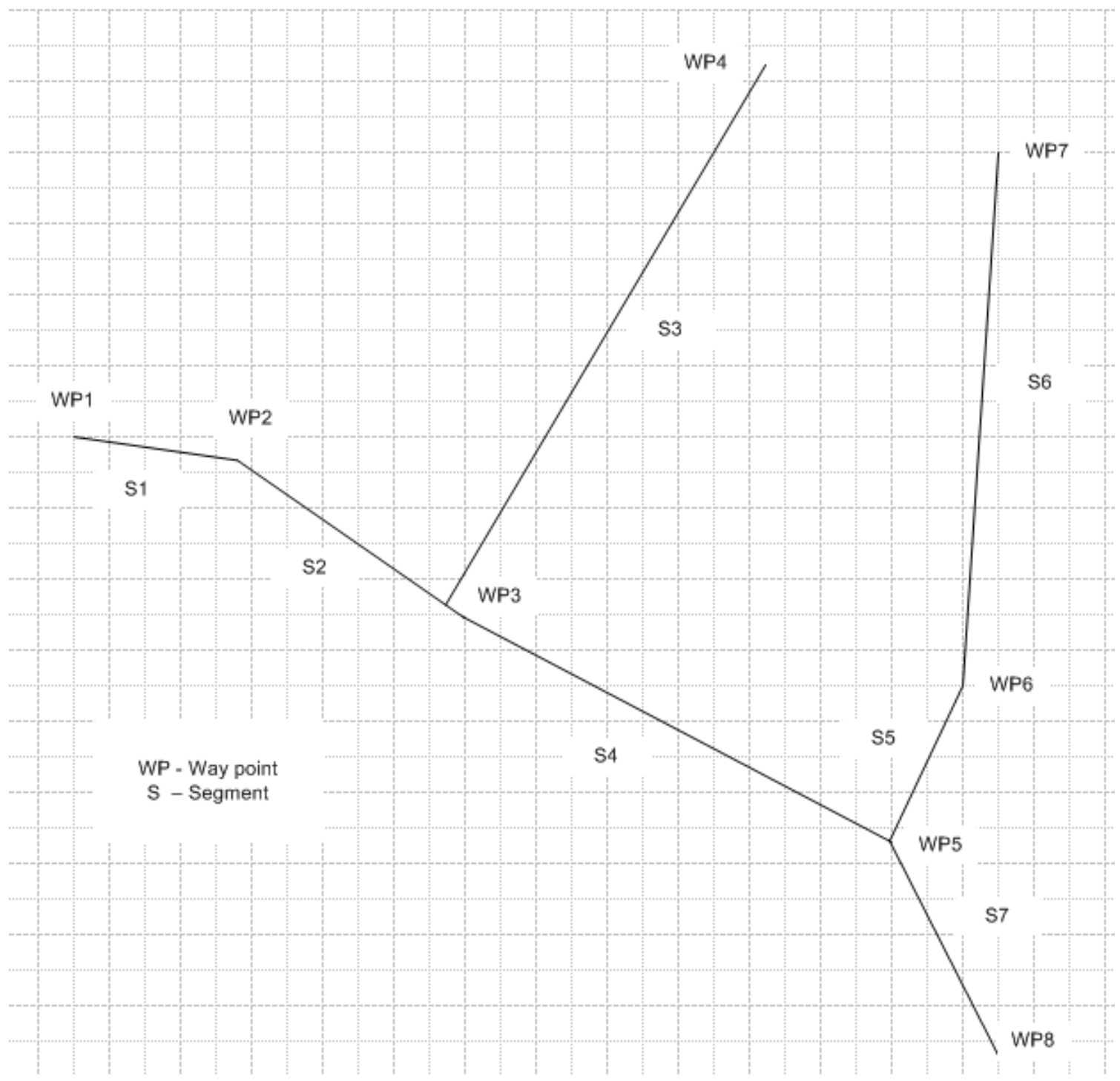

Figure 4.12: Path map with segments and way points 
The data set selected for the testing purpose has measurements spanning around 33 hours. Figure 4.13 shows the tracks from the 25 targets over the time with prediction algorithm. Here prediction is carried out every $300 \mathrm{~s}$ which can be changed according to the requirement. Figure 4.14 shows the tracks from the interested two targets where the first target was moving in Segment 3 in the direction of way-point 4 and the second target is in segment 2 and moving towards way-point 3. Above two targets moved over $1000 \mathrm{~km}$ and $1200 \mathrm{~km}$ in our local X-Y framework and generated four and three measurements respectively during the above time interval.

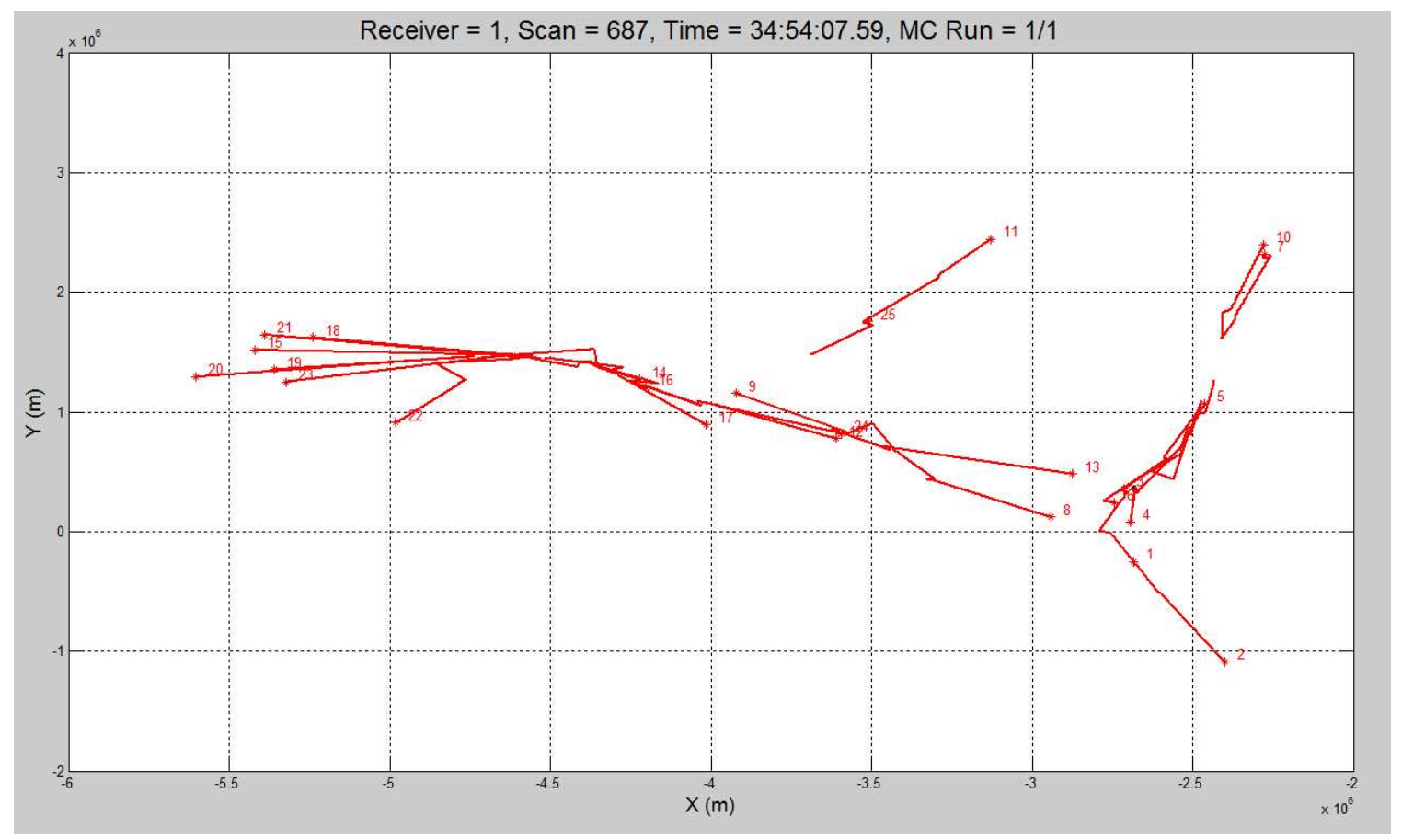

Figure 4.13: Tracks from 25 targets 


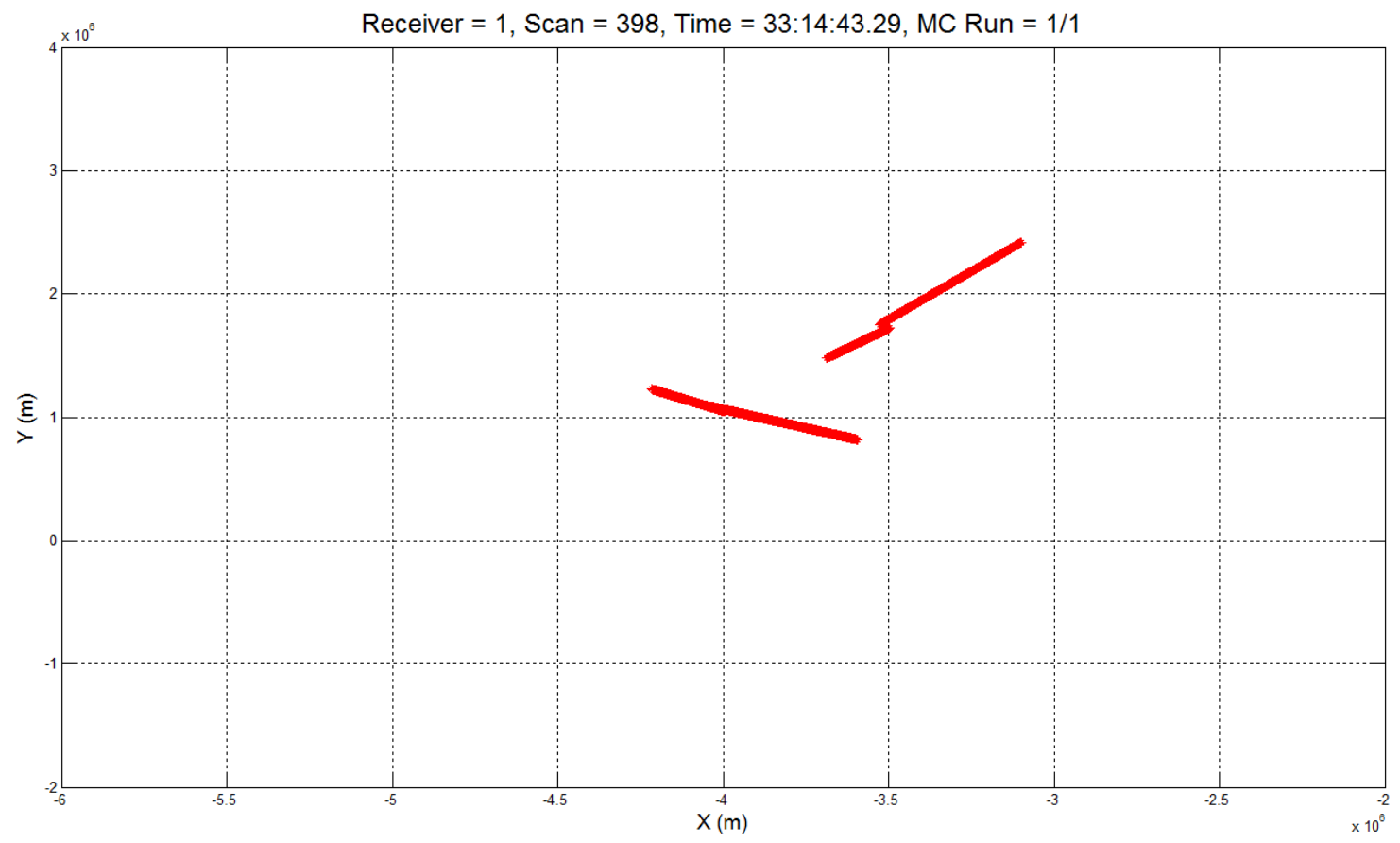

Figure 4.14: Tracks from prediction

The tracks from the above test (Figure 4.14) is tested with retrodiction algorithm and Figure 4.15 shows the retrodicted tracks. 


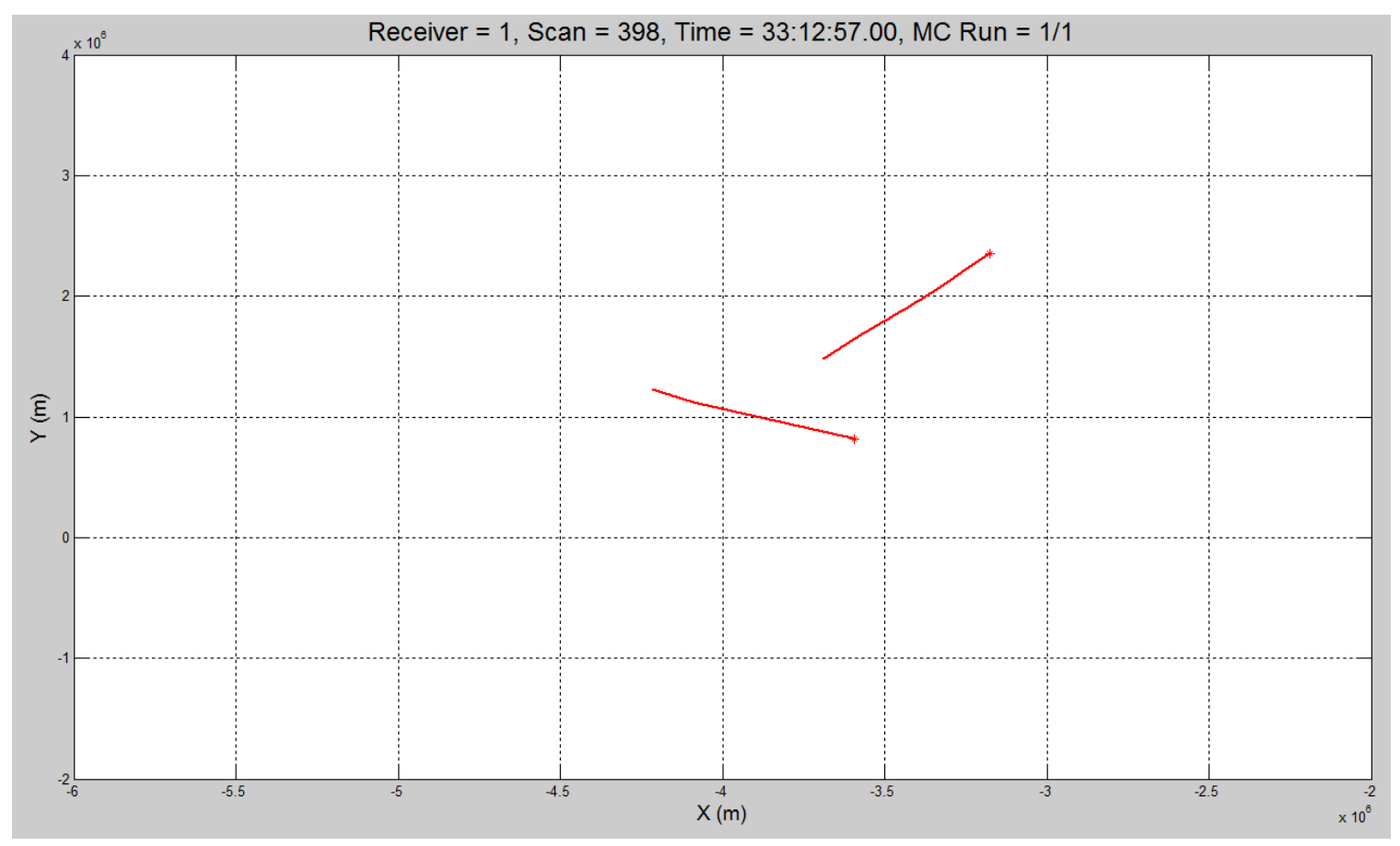

Figure 4.15: Tracks from retrodiction

To get the root mean square error (RMSE) for position estimate of our prediction and retrodiction algorithm, truth file for the above scenario is required. But in real scenarios it is not possible to get the truth values. So retrodicted tracks are kept as truth and RMSE test is carried out. Figure 4.16 shows the RMS position errors for both targets. It can be seen that, when the measurements are received the position RMSE is reduced and on average the position RMSE is around $17 \mathrm{~km}$. But when considering the distances these targets traveled and given only seven measurements received over this 34 hour period time, this value can be expected. 


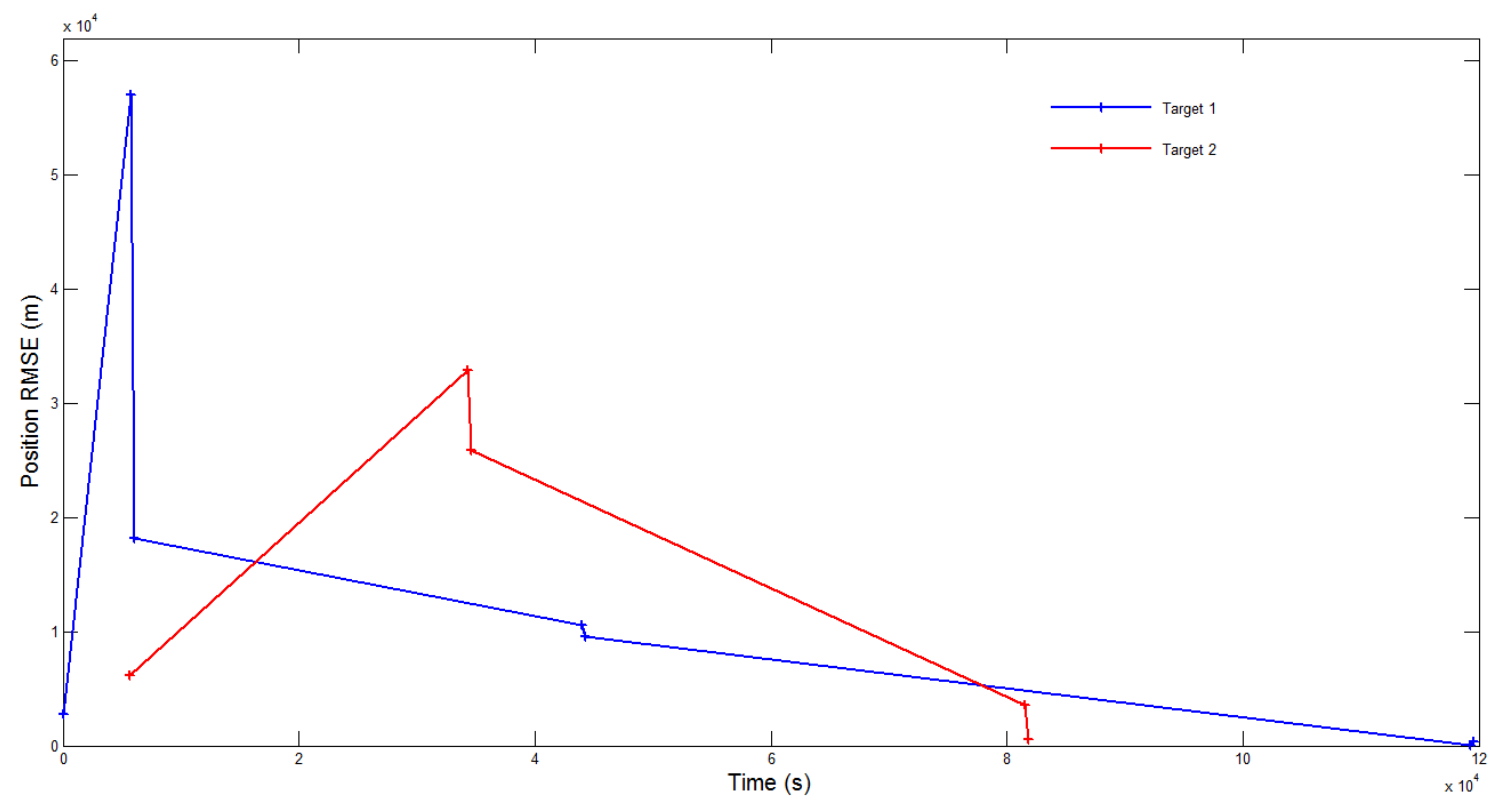

Figure 4.16: RMS position errors comparison 


\section{Chapter 5}

\section{Conclusions and Future Work}

\subsection{Conclusions}

In this thesis, new tracking and retrodiction algorithms for path constrained targets are presented. As a target moves along a constrained path with different segment constraints, measurements are updated by sensor with low revisit rate. But target changes its motion due to segment constraints in between successive measurements. This results in a poor performance of existing algorithms due to not incorporating the path map information. The proposed tracking algorithm uses a prediction technique considering the segment change and the time taken to reach a junction. During prediction near the junctions, possible velocity change also considered for each segment to give a better estimate. A retrodiction algorithm considering the path map also developed in this paper based on (Rauch et al., 1965) and (Koch, 2000). The retrodiction algorithm developed here supports the segment change and associated motion model changes in a junction. 
The results shows that the proposed algorithm in tracking and retrodiction performs better compared to the other algorithms in literature. The better performance of tracking algorithm is due to the prediction algorithm, which considers the path map information. Further already existing retrodiction algorithms does not consider the segment change explicitly as in the proposed retrodiction algorithm, resulting in a lower RMS error in position and velocity during retrodiction compared to the existing algorithms.

\section{$5.2 \quad$ Future Work}

The proposed algorithms could be extended to data association problems where the assumptions of zero false alarms and unity probability of detection can be relaxed. Further uncertainty in way-points and segments can also be included in path map information. Incorporating the above uncertainty information will increase the usefulness of the proposed algorithms in real data applications. Finally building the path map information automatically from the real data would be a great area to explore. It will take the applications of proposed prediction and retrodiction algorithms to the next level. 


\section{Appendix A}

\section{Algorithm Derivations}

\section{A.0.1 Filtering Algorithm Derivation}

Derivation for path constrained target estimator is given here.

$$
\left.P\left[x(k) \mid Z_{1}^{k}\right]=\sum_{M_{j^{\prime}}, M_{i^{\prime}} \in A(k)} P\left[x(k) \mid M_{j^{\prime}}, M_{i^{\prime}}, Z_{1}^{k}\right)\right] P\left[M_{j^{\prime}}, M_{i^{\prime}} \mid Z_{1}^{k}\right]
$$

consider

$$
\left.P\left[x(k) \mid M_{j^{\prime}}, M_{i^{\prime}}, Z_{1}^{k}\right)\right]=\frac{P\left[Z(k) \mid x(k), M_{j^{\prime}}, M_{i^{\prime}}\right] P\left[x(k) \mid M_{j^{\prime}}, M_{i^{\prime}}, Z_{1}^{k-1}\right]}{P\left[z(k) \mid M_{j^{\prime}}, M_{i^{\prime}}, Z_{1}^{k-1}\right]}
$$

where $P\left[z(k) \mid M_{j^{\prime}}, M_{i^{\prime}}, Z_{1}^{k-1}\right]$ is the likelihood and $P\left[x(k) \mid M_{j^{\prime}}, M_{i^{\prime}}, Z_{1}^{k-1}\right]$ is the prior. The prior can be extended as follows

$$
P\left[x(k) \mid M_{j^{\prime}}, M_{i^{\prime}}, Z_{1}^{k-1}\right]=P\left[x(k) \mid x(k-\tau), M_{j^{\prime}}, M_{i^{\prime}}, Z_{1}^{k-1}\right] P\left[x(k-\tau) \mid M_{j^{\prime}}, M_{i^{\prime}}, Z_{1}^{k-1}\right]
$$


Consider the second term in the prior

$$
P\left[x(k-\tau) \mid M_{j^{\prime}}, M_{i^{\prime}}, Z_{1}^{k-1}\right]=P\left[x(k-\tau) \mid M_{i^{\prime}}, Z_{1}^{k-1}\right]
$$

which can be written according to (Bar-Shalom et al., 2001) as

$$
\begin{aligned}
P\left[x(k-\tau) \mid M_{i^{\prime}}, Z_{1}^{k-1}\right]= & \mathcal{N}\left(\left(x(k-\tau) ; E\left[x(k-\tau) \mid M_{i^{\prime}},\right.\right.\right. \\
& \left.\left.\sum_{M_{i} \in A(k-1)} \hat{x}^{i}(k-1 \mid k-1) \mu_{i \mid i^{\prime}}(k-1 \mid k-1)\right], \operatorname{Cov}[.]\right)
\end{aligned}
$$

where

$$
\mu_{i \mid i^{\prime}}(k-1 \mid k-1)=P\left\{M_{i} \mid M_{i^{\prime}}, Z_{1}^{k-1}\right\}
$$

Now consider the first term in the prior

$$
P\left[x(k) \mid x(k-\tau), M_{j^{\prime}}, M_{i^{\prime}}, Z_{1}^{k-1}\right]=P\left[x(k) \mid x(k-\tau), M_{j^{\prime}}\right]
$$

Now consider the following

$$
\begin{aligned}
\left.P\left[M_{i^{\prime}}, M_{j^{\prime}} \mid Z_{1}^{k}\right)\right] & \left.=P\left[M_{i^{\prime}}, M_{j^{\prime}} \mid z(k), Z_{1}^{k-1}\right)\right] \\
& =\frac{1}{C_{i^{\prime} j^{\prime}}} P\left[z(k) \mid M_{j^{\prime}}, M_{i^{\prime}}, Z_{1}^{k-1}\right] P\left[M_{j^{\prime}} \mid M_{i}^{\prime}, Z_{1}^{k-1}\right] P\left[M_{i}^{\prime} \mid Z_{1}^{k-1}\right] \\
& =\frac{1}{C_{i^{\prime} j^{\prime}}} \Lambda_{i^{\prime} j^{\prime}}(k)\left[p_{i^{\prime} j^{\prime}}\left(S_{k-1}, S_{k}\right)\right] \mu_{i^{\prime}}(k-\tau)
\end{aligned}
$$


where

$$
\begin{aligned}
\mu_{i^{\prime}}(k-\tau) & =P\left\{M_{i^{\prime}} \mid Z_{1}^{k-1}\right\} \\
& =\sum_{M_{i} \in A(k-1)} P\left\{M_{i^{\prime}} \mid M_{i}, Z_{1}^{k-1}\right\} P\left\{M_{i} \mid Z_{1}^{k-1}\right\} \\
& =\sum_{M_{i} \in A(k-1)}\left[p_{i i^{\prime}}\left(S_{k-1}\right)\right] \mu_{i}(k-1)
\end{aligned}
$$

\section{A.0.2 Retrodiction Algorithm Derivation}

This part presents algorithm derivation of retrodiction for variable structure IMM without any constraints. This is similar to the algorithm proposed in (Nandakumaran et al., 2009) but differs in mode matched retrodiction.

Let $A(t)=\left(M_{1}(t), \ldots, M_{u}(t), \ldots\right)$ and $A(t+1)=\left(M_{1}(t+1), \ldots, M_{v}(t+1), \ldots\right)$

Smoothed density $P\left[x(t) \mid Z_{1}^{k}\right]$ is required where $t<k$. It can be written as

$$
P\left[x(t) \mid Z_{1}^{k}\right]=\sum_{M_{u} \in A(t)} P\left[x(t) \mid M_{u}, Z_{1}^{k}\right] P\left[M_{u} \mid Z_{1}^{k}\right]
$$

The first term in (A.10) can be written as

$$
P\left[x(t) \mid M_{u}, Z_{1}^{k}\right]=\sum_{M_{v} \in A(t+1)} P\left[x(t) \mid M_{u}, M_{v}, Z_{1}^{k}\right] P\left[M_{v} \mid M_{u}, Z_{1}^{k}\right]
$$

Using the total probability theorem and the Bayes' rule the density $P\left[x(t) \mid M_{u}, Z_{1}^{k}\right]$ can be simplified as follows 


$$
\begin{aligned}
P\left[x(t) \mid M_{u}, M_{v}, Z_{1}^{k}\right] & =\int P\left[x(t), x(t+1) \mid M_{u}, M_{v}, Z_{1}^{k}\right] d x_{t+1} \\
& =\int P\left[x(t) \mid x(t+1), M_{u}, M_{v}, Z_{1}^{k}\right] P\left[x(t+1) \mid M_{u}, M_{v}, Z_{1}^{k}\right] d x_{t+1} \\
& =\int P\left[x(t) \mid x(t+1), M_{u}, M_{v}, Z_{1}^{t}\right] P\left[x(t+1) \mid M_{v}, Z_{1}^{k}\right] d x_{t+1} \\
& =\int \frac{P\left[x(t+1) \mid x(t), M_{u}, M_{v}, Z_{1}^{t}\right] P\left[x(t) \mid M_{u}, Z_{1}^{t}\right]}{P\left[x(t+1) \mid M_{u}, M_{v}, Z_{1}^{t}\right]} \\
& P\left[x(t+1) \mid M_{v}, Z_{1}^{k}\right] d x_{t+1}
\end{aligned}
$$

In the above derivation, $P\left[x(t) \mid x(t+1), M_{u}, M_{v}, Z_{1}^{k}\right]$ is replaced with $P[x(t) \mid x(t+$ 1), $\left.M_{u}, M_{v}, Z_{1}^{t}\right]$ and $P\left[x(t) \mid M_{u}, Z_{1}^{t}\right]$ is the filtered mode conditioned density. The term $P\left[x(t+1) \mid M_{v}, Z_{1}^{k}\right]$ is the smoothed density at time $(t+1)$ given the mode $M_{v}$ and the term in the denominator is constant. Using the Gaussian distribution computation rules, we can write $P\left[x(t+1) \mid x(t), M_{u}, M_{v}, Z_{1}^{t}\right] P\left[x(t) \mid M_{u}, Z_{1}^{t}\right]$ in (A.15) as follows

$$
\begin{aligned}
& P\left[x(t+1) \mid x(t), M_{u}, M_{v}, Z_{1}^{t}\right] P\left[x(t) \mid M_{u}, Z_{1}^{t}\right] \\
& =P\left[x(t+1), x(t) \mid M_{u}, M_{v}, Z_{1}^{t}\right] \\
& =\mathcal{N}\left(\left[\begin{array}{c}
x(t) \\
x(t+1)
\end{array}\right] \mid\left[\begin{array}{c}
\hat{x}^{u}(t \mid t) \\
F^{v}(t+1, t)
\end{array}\right]\right. \\
& {\left[\begin{array}{c}
\hat{x}^{u}(t \mid t)
\end{array}\right],} \\
& P^{u}(t \mid t) \\
& P^{u}(t \mid t) F^{v}(t+1, t)
\end{aligned}
$$


In (A.14) now consider the first term

$$
P\left[x(t) \mid x(t+1), M_{u}, M_{v}, Z_{1}^{t}\right]=\mathcal{N}(x(t) \mid m, P)
$$

where

$$
\begin{array}{r}
m=\hat{x}^{u}(t \mid t)+A\left(x(t+1)-F^{v}(t+1, t) \hat{x}^{u}(t \mid t)\right) \\
P=P^{u}(t \mid t)-A\left[F^{v}(t+1, t) P^{u}(t \mid t) F^{v}(t+1, t)^{T}+Q_{v}(t \mid t)\right] A^{T}
\end{array}
$$

where

$$
A=P^{u}(t \mid t) F^{v}(t+1, t)^{T}\left[F^{v}(t+1, t) P^{u}(t \mid t) F^{v}(t+1, t)^{T}+Q_{v}(t \mid t)\right]^{-1}
$$

Now from (A.12), the joint distribution of $x(t)$ and $x(t+1)$ given all the measurement is

$$
\begin{aligned}
& P\left[x(t), x(t+1) \mid M_{u}, M_{v}, Z_{1}^{k}\right] \\
& =P\left[x(t) \mid x(t+1), M_{u}, M_{v}, Z_{1}^{k}\right] P\left[x(t+1) \mid M_{u}, M_{v}, Z_{1}^{k}\right] \\
& =\mathcal{N}(x(t) \mid m, P) \mathcal{N}\left(x(t+1) \mid \hat{x}^{v}(t+1 \mid k), P^{v}(t+1 \mid k)\right) \\
& =\mathcal{N}\left(\left[\begin{array}{c}
x(t+1) \\
x(t)
\end{array}\right] \mid\left[\begin{array}{c}
\hat{x}^{v}(t+1 \mid k) \\
\hat{x}^{u}(t \mid t)+A\left(\hat{x}^{v}(t+1 \mid k)-F^{v}(t+1, t) \hat{x}^{u}(t \mid t)\right)
\end{array}\right],\right. \\
& \left.\left[\begin{array}{cc}
P^{v}(t+1 \mid k) & P^{v}(t+1 \mid k) A^{T} \\
A P^{v}(t+1 \mid k) & A P A^{T}+P
\end{array}\right]\right)
\end{aligned}
$$

So based on the above derivation, mode matched smoothing mean and covariance 
are given by (Rauch et al., 1965)

$$
\begin{aligned}
& \hat{x}^{u v}(t \mid k)=\hat{x}^{u}(t \mid t)+A\left[\hat{x}^{v}(t+1 \mid k)-\hat{x}^{u v}(t+1 \mid t)\right] \\
& P^{u v}(t \mid k)=P^{u}(t \mid t)+A\left[P^{v}(t+1 \mid k)-P^{u v}(t+1 \mid t)\right] A^{T}
\end{aligned}
$$

where $A=P^{u}(t \mid t) F^{v}(t+1)^{T}\left[F^{v}(t+1) P^{u}(t \mid t) F^{v}(t+1)^{T}+Q^{v}(t+1)\right]^{-1}$. Further the terms $\hat{x}^{u v}(t+1 \mid t)$ and $\hat{P}^{u v}(t+1 \mid t)$ are written as

$$
\begin{aligned}
\hat{x}^{u v}(t+1 \mid t) & =F^{v}(t+1) \hat{x}^{u}(t \mid t) \\
P^{u v}(t \mid k) & =F^{v}(t+1) P^{u}(t \mid t) F^{v}(t+1)^{T}+Q^{v}(t+1)
\end{aligned}
$$

All other derivations are same as in (Nandakumaran et al., 2009) except the derivation for $P\left[M_{u} \mid Z_{1}^{k}\right]$ which can be written as

$$
\begin{aligned}
P\left[M_{u} \mid Z_{1}^{k}\right] & \simeq P\left(M_{u} \mid \Upsilon_{t+1 \mid k}, Z_{1}^{t}\right) \\
& =\frac{1}{f} P\left(\Upsilon_{t+1 \mid k} \mid M_{u}, Z_{1}^{t}\right) P\left(M_{u} \mid Z_{1}^{t}\right)
\end{aligned}
$$

where $\Upsilon_{t+1 \mid k}$ is sufficient statistic for the measurement set $Z_{t+1}^{k}$. The normalizing constant is given by

$$
f=\sum_{M_{u} \in A(t)} P\left(\Upsilon_{t+1 \mid k} \mid M_{u}, Z_{1}^{t}\right) P\left(M_{u} \mid Z_{1}^{t}\right)
$$




$$
\begin{aligned}
& P\left(\Upsilon_{t+1 \mid k} \mid M_{u}, Z_{1}^{t}\right) \text { in }(\text { A.29) can be written as } \\
& \begin{aligned}
P\left(\Upsilon_{t+1 \mid k} \mid M_{u}, Z_{1}^{t}\right) & =\sum_{M_{v} \in A(t+1)} P\left[\left(\Upsilon_{t+1 \mid k} \mid M_{v}, M_{u}, Z_{1}^{t}\right] P\left[M_{v} \mid M_{u}, Z_{1}^{t}\right]\right. \\
& =\sum_{M_{v} \in A(t+1)} P\left[\left(\Upsilon_{t+1 \mid k} \mid \Upsilon_{t \mid t}, M_{v}, M_{u}\right]\left[M_{v} \mid M_{u}\right]\right. \\
& =\sum_{M_{v} \in A(t+1)} p_{u v} \mathcal{N}\left(\Upsilon_{t+1 \mid k} \mid \Upsilon_{t \mid t}\right)
\end{aligned}
\end{aligned}
$$

\section{A.0.3 Retrodiction Algorithm Derivation for Path Constrained Targets}

The above derivation is extended to include the path segment change during retrodiction process as follows. Let $A(t)=\left(M_{1}(t), M_{2}(t), \ldots, M_{u}(t), \ldots\right)$ is the mode set in the time interval $(t-1, t] . t+\delta$ is the time at which target changes the segment where $0<\delta<1$. Further denote the mode in effect during the time interval $(t, t+\delta]$ as $M_{u^{\prime}}$ and during $(t+\delta, t+1]$ as $M_{v^{\prime}}$ where $M_{u^{\prime}}, M_{v^{\prime}} \in A(t+1)$

$$
P\left[x(t) \mid Z_{1}^{k}\right]=\sum_{\forall M_{u}} P\left[x(t) \mid M_{u}, Z_{1}^{k}\right] P\left[M_{u} \mid Z_{1}^{k}\right]
$$

The first term in (A.35) can be written as

$$
P\left[x(t) \mid M_{u}, Z_{1}^{k}\right]=\sum_{M_{u^{\prime}} M_{v^{\prime}} \in A(t+1)} P\left[x(t) \mid M_{u}, M_{u^{\prime}}, M_{v^{\prime}}, Z_{1}^{k}\right] P\left[M_{u^{\prime}}, M_{v^{\prime}} \mid M_{u}, Z_{1}^{k}\right]
$$


we can write $P\left[x(t) \mid M_{u}, M_{u^{\prime}}, M_{v^{\prime}}, Z_{1}^{k}\right]$ as follows

$$
\begin{gathered}
P\left[x(t) \mid M_{u}, M_{u^{\prime}}, M_{v^{\prime}}, Z_{1}^{k}\right]=\int \frac{P\left[x(t+1) \mid x(t), M_{u}, M_{u^{\prime}}, M_{v^{\prime}}, Z_{1}^{t}\right] P\left[x(t) \mid M_{u}, Z_{1}^{t}\right]}{P\left[x(t+1) \mid M_{u}, M_{u^{\prime}}, M_{v^{\prime}}, Z_{1}^{t}\right]} \\
P\left[x(t+1) \mid M_{u^{\prime}}, M_{v^{\prime}}, Z_{1}^{k}\right] d x_{t+1}
\end{gathered}
$$

Based on (A.37), mode matched filtering equation can be written as follows

$$
\begin{aligned}
& \hat{x}^{u\left(u^{\prime} v^{\prime}\right)}(t \mid k)=\hat{x}^{u}(t \mid t)+A\left[\hat{x}^{u^{\prime} v^{\prime}}(t+1 \mid k)-\hat{x}^{u\left(u^{\prime} v^{\prime}\right)}(t+1 \mid t)\right] \\
& P^{u\left(u^{\prime} v^{\prime}\right)}(t \mid k)=P^{u}(t \mid t)+A\left[P^{u^{\prime} v}(t+1 \mid k)-P^{u\left(u^{\prime} v^{\prime}\right)}(t+1 \mid t)\right] A^{T}
\end{aligned}
$$

where $\left.A=P^{u}(t \mid t)\left\{F^{v^{\prime}}(t+1, t+\delta) R F^{u^{\prime}}(t+\delta, t)\right]\right\}^{T}\left[P^{u\left(u^{\prime} v^{\prime}\right)}(t+1 \mid t)\right]^{-1}$. Further the terms $\hat{x}^{u v}(t+1 \mid t)$ and $P^{u v}(t+1 \mid t)$ can be derived as

$$
\begin{aligned}
\hat{x}^{u\left(u^{\prime} v^{\prime}\right)}(t+1 \mid t) & =F^{v^{\prime}}(t+1, t+\delta) R F^{u^{\prime}}(t+\delta, t) \hat{x}^{u}(t \mid t) \\
P^{u\left(u^{\prime} v^{\prime}\right)}(t+1 \mid t) & =F^{v^{\prime}}(t+1, t+\delta) R\left[F^{u^{\prime}}(t+\delta, t) P^{u}(t \mid t) F^{u^{\prime}}(t+\delta, t)^{T}\right. \\
& \left.+Q^{u^{\prime}}(t+\delta, t)\right] R^{T} F^{v^{\prime}}(t+1, t+\delta)^{T}+Q^{v^{\prime}}(t+\delta, t+1)
\end{aligned}
$$




\section{Bibliography}

Arulampalam, M. S., Gordon, N., Orton, M., and Ristic, B. (2002). A variable structure multiple model particle filter for GMTI tracking. Proc. of the Fifth International Conference on Information Fusion, 2, 927-934.

Bar-Shalom, Y. and Li, X. R. (1995). Multitarget-Multisensor Tracking: Principles and Techniques. YBS Publishing.

Bar-Shalom, Y., Li, X. R., and Kirubarajan, T. (2001). Estimation with Applications to Tracking and Navigation. YBS Publishing.

Blom, H. A. P. and Bar-Shalom, Y. (1988). The interacting multiple model algorithm for systems with Markovian switching coefficients. IEEE Trans. Automatic Control, 33, 780-783.

Deisenroth, M. P. and Ohlsson, H. (2011). A general perspective on Gaussian filtering and smoothing: Explaining current and deriving new algorithms. American Control Conference, 36, 1807-1812.

Fraser, D. and Potter, J. E. (1969). The Optimum Linear Smoother as a Combination of Two Optimum Linear Filters. IEEE Trans. on Automatic control, pages 387-390. 
Helmick, R. E., Blair, W. D., and Hoffman, S. A. (1994). One-step fixed-lag smoothers for Markovian switching systems. American Control Conference, 1, 782-786.

Helmick, R. E., Blair, W. D., and Hoffman, S. A. (1995). Fixed-interval smoothing for Markovian switching systems. IEEE Transactions on Information Theory, 41, $1845-1855$.

Hernandez, M. L. (2003). Performance Bounds for GMTI Tracking. Proc. of the International Conference on Information Fusion, 1, 406-413.

Hernandez, M. L., Kirubarajan, T., and Bar-Shalom, Y. (2004). Multisensor Resource Deployment Using Posterior Cramer-Rao Bounds. IEEE Trans. on Aerospace and Electronic Systems, 40, 399-416.

Hernandez, M. L., Ristic, B., Farina, A., Sathyan, T., and Kirubarajan, T. (2008). Performance measure for Markovian switching systems using best-fitting Gaussian distributions. IEEE Trans. on Aerospace and Electronic Systems, 44, 724-747.

Horridge, P. R. and Hernandez, M. L. (2003). Performance Bounds for Angle-Only Filtering with Application to Sensor Network Management. Proc. of the International Conference on Information Fusion.

Kirubarajan, T., Bar-Shalom, Y., Pattipati, K. R., and Kadar, I. (2000). Ground Target Tracking with Topography-Based Variable Structure IMM Estimator. IEEE Trans. Aerospace and Electronic Systems, 36, 26-46.

Koch, W. (2000). Fixed-interval retrodiction approach to Bayesian IMM-MHT for maneuvering multiple targets. IEEE Trans. Aerospace and Electronic Systems, $\mathbf{3 6}$, $2-14$. 
Kyriakides, I., Trueblood, T., Morrell, D., and Papandreou-Suppappola, A. (2008). Multiple target tracking using particle filtering and adaptive waveform design. Asilomar Conference on Signals, Systems and Computers, pages 1188-1192.

Li, X. R. and Bar-Shalom, Y. (1996). Multiple-model estimation with variable structure. IEEE Transactions on Automatic Control, 41, 478-493.

Nandakumaran, N., Lang, T., Mcdonald, M., and Kirubarajan, T. (2009). IMM Forward Filtering and Backward Smoothing for Maneuvering Target Tracking. Proc. of Signal and Data Processing of Small Targets, 7445.

Pannetier, B., Benameur, K., Nimier, V., and Rombaut, M. (2005). Ground moving target tracking with road constraint. International Conference on Information Fusion, 1, 138-149.

Rauch, H. E., Tung, F., and Striebel, C. T. (1965). Maximum likelihood estimates of linear dynamic systems. The American Institute of Aeronautics and Astronautics, 3, 1445-1450.

Shea, P. J., Zadra, T., Klamer, D. M., Frangione, E., and Brouillards, R. (2000). Improved state estimation through use of roads in ground tracking. In Proc. of Signal and Data Processing of Small Targets, 4048.

Simandl, M., Kralovec, J., and Tichavsky, P. (2001). Filtering, Predictive, and Smoothing Cramer-Rao Bounds for discrete-Time Nonlinear Dynamic Systems. Proc. of the International Conference on Information Fusion, 37, 1703-1716.

Streller, D. (2008). Road map assisted ground target tracking. International Conference on Information Fusion. 
Tugnait, J. K. (2000). Interacting multiple model fixed-lag smoothing algorithm for Markovian switching systems. IEEE Trans. on Aerospace and Electronic Systems, 36, 243-250.

Ulmke, M. and Koch, W. (2006). Road-map assisted ground moving target tracking. IEEE Transactions on Aerospace and Electronic Systems, 42, 1264-1274.

Yeddanapudi, M., Bar-Shalom, Y., and Pattipati, K. (1997). IMM estimation for multitarget-multisensor air traffic surveillance. Proc. of the IEEE, 85, 80-96. 